\title{
CUBESAT CONSTELLATION ANALYSIS FOR DATA RELAYING
}

\author{
A Thesis \\ presented to \\ the Faculty of California Polytechnic State University, \\ San Luis Obispo
}

\author{
In Partial Fulfillment \\ of the Requirements for the Degree \\ Master of Science in Aerospace Engineering
}

by

Bradley Ryan Smalarz

December 2011 
(C) 2011

Bradley Ryan Smalarz

ALL RIGHTS RESERVED 


\section{COMMITTEE MEMBERSHIP}

TITLE:

AUTHOR:

DATE SUBMITTED:

COMMITTEE CHAIR:

COMMITTEE MEMBER:

COMMITTEE MEMBER:

COMMITTEE MEMBER:
CubeSat Constellation Analysis for Data Relaying

Bradley Ryan Smalarz

December 2011
Dr. Eric Mehiel, Aerospace Engineering Department Chair

Dr. Kira Abercrombie, Aerospace Engineering Professor
Dr. Jeffery Puschell, Principal Engineering Fellow, Raytheon Company

David Esposto, Aerospace Engineering Department

Lecturer 


\section{ABSTRACT \\ CubeSat Constellation Analysis for Data Relaying \\ Bradley Ryan Smalarz}

Current CubeSat communication technology limits the amount of time, and number of accesses with ground stations. It has been proposed to use a constellation of CubeSats to improve relay performance and increase the number of accesses between a CubeSat and ground stations. By using the spatial and temporal analysis features of STK, coupled with the STK/Matlab interface a robust tool was created to analyze the performance of CubeSat constellations based on a store-and-forward communications model which is not currently supported by the STK Engine. Utilizing the Connect messaging format through a socket connection on the local machine, a Matlab graphical user interface, called SATCAT, was constructed in order to provide a user with the ability to control many aspects of the STK Engine externally. A function was created to use three Time Ordered Access (TOA) reports from STK to determine how long it would take for data to be relayed from a target to a ground station through a constellation of CubeSats. Three sample scenarios were created to demonstrate the use and performance analysis capabilities of SATCAT. The performance of a single CubeSat was analyzed and compared to the performance of a three CubeSat constellation and a thirty-seven CubeSat constellation. It was shown that a constellation of three CubeSats decreased the average relay time from 328 minutes to 149 minutes and a constellation of thirty-seven CubeSats further reduced the average relay time to only 3 minutes. While decreasing the average relay time, the constellation of three CubeSats also increased the number of accesses over a twenty-four hour period from 6 to 36, and the constellation of thirty-seven CubeSats allowed for 564 accesses. 


\section{ACKNOWLEDGEMENTS}

The author of this paper would like to thank Analytical Graphics, Inc. for the educational license of their System Tool Kit software used extensively in this project.

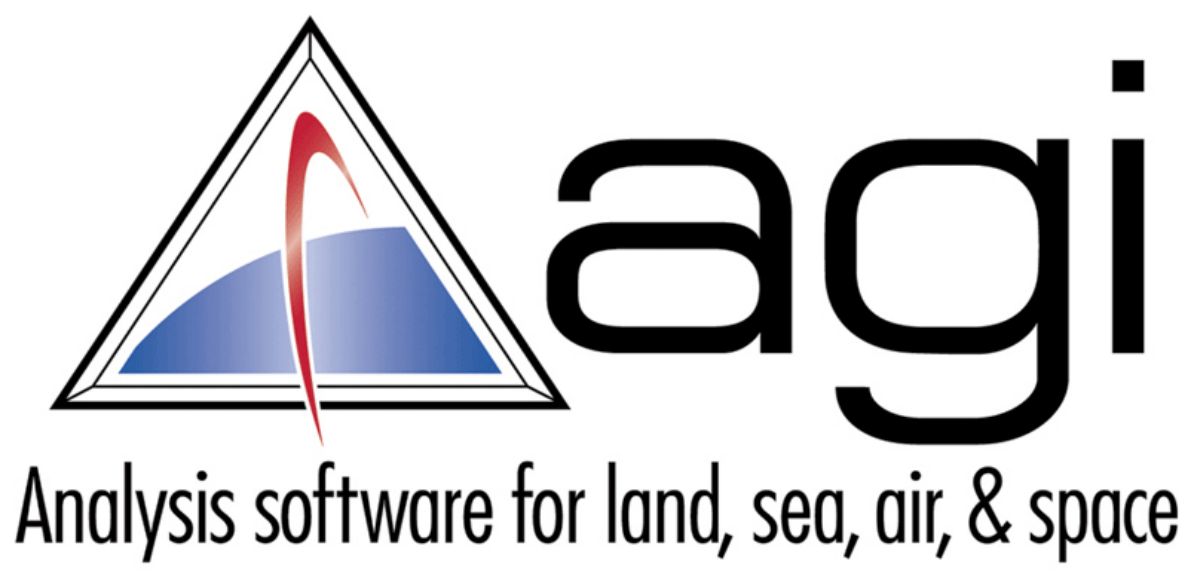




\section{Table of Contents}

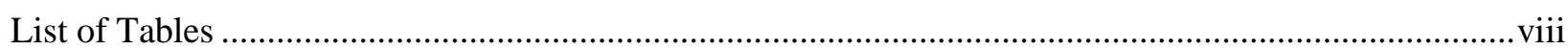

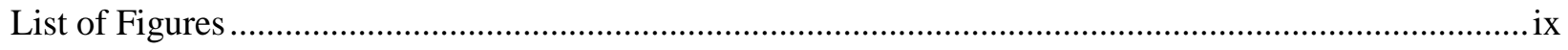

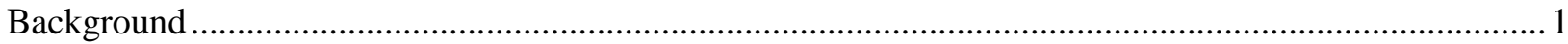

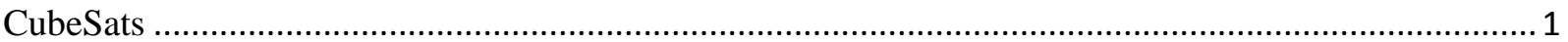

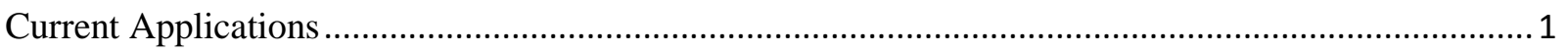

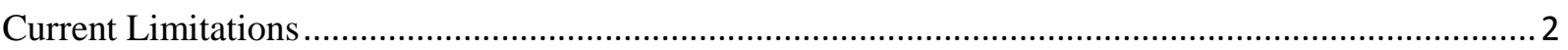

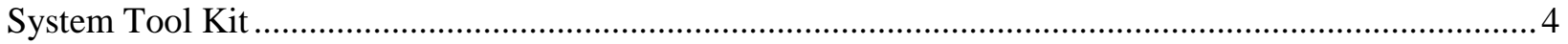

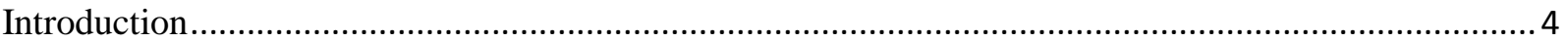

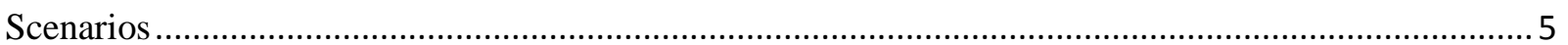

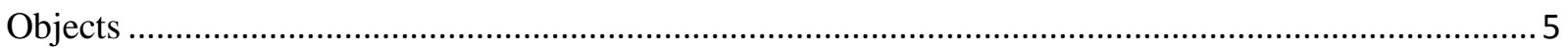

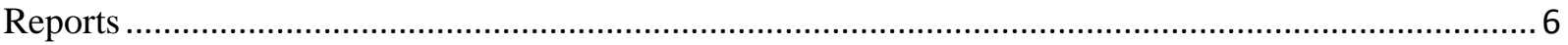

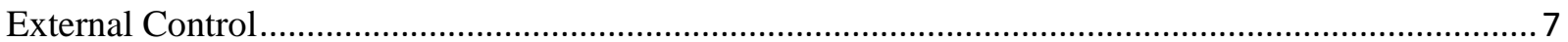

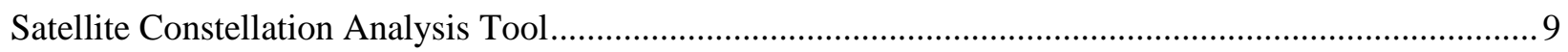

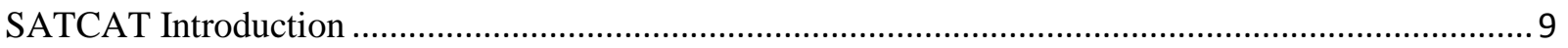

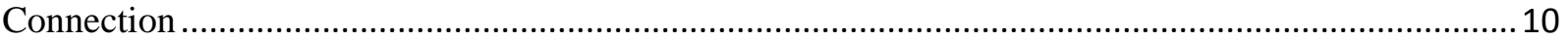

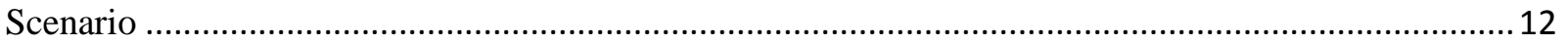

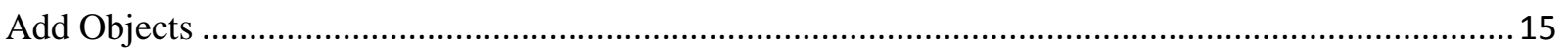

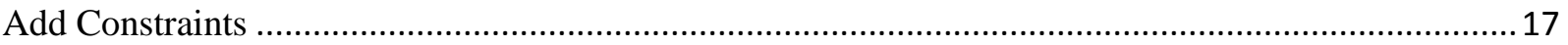

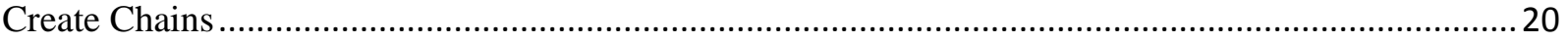

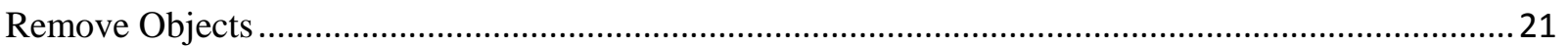

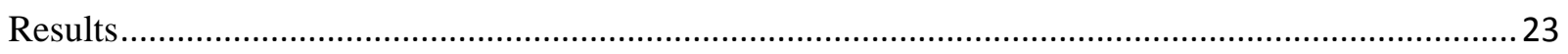

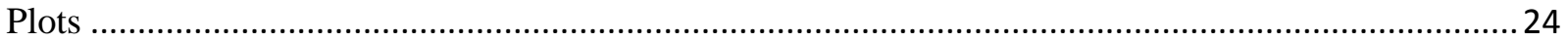

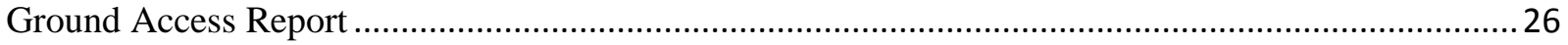

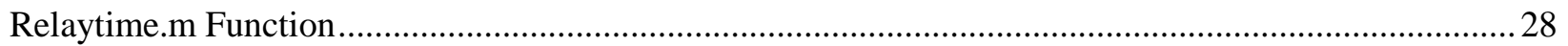

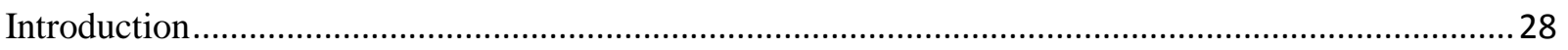

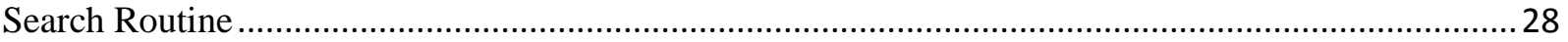

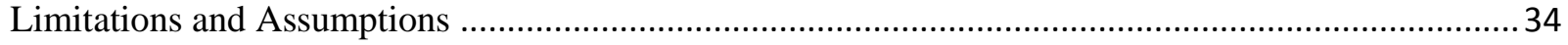

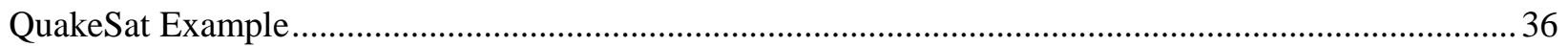

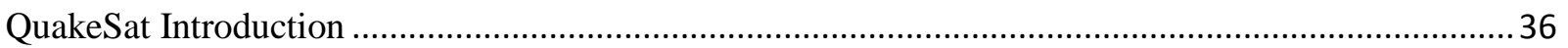

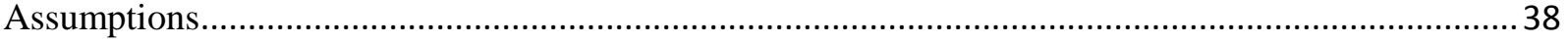




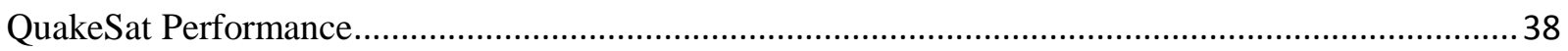

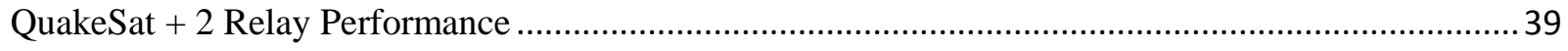

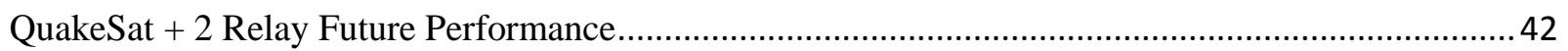

QuakeSat + Constellation Performance …............................................................................. 42

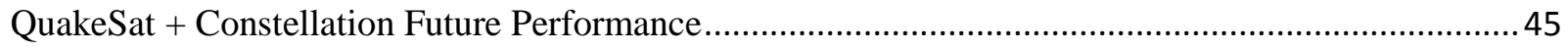

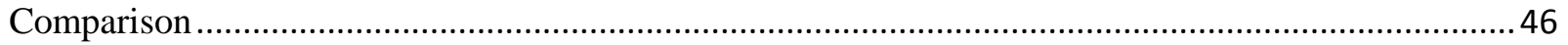

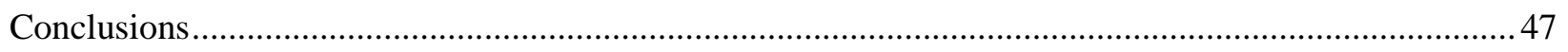

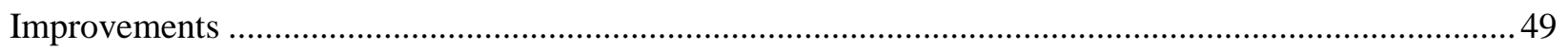

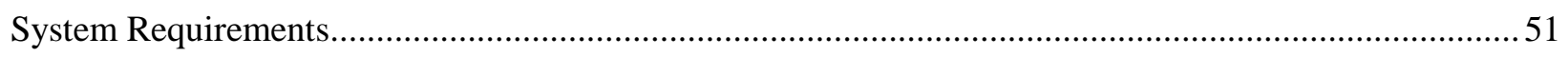

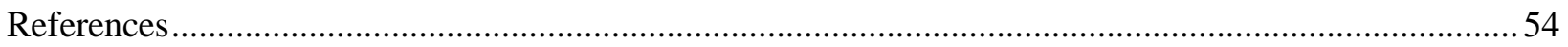




\section{List of Tables}

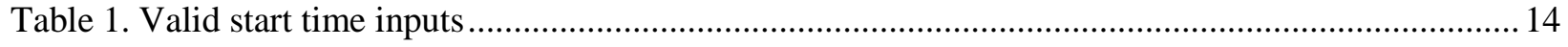

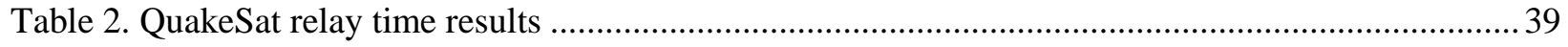

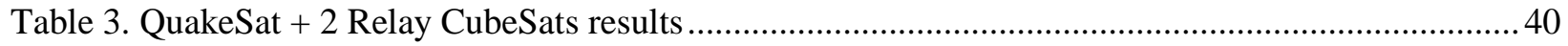

Table 4. QuakeSat + 2 Relays results one year in the future ..............................................................42

Table 5. QuakeSat + Constellation relay time results ....................................................................... 43

Table 6. QuakeSat + Constellation relay times a year later ............................................................... 45

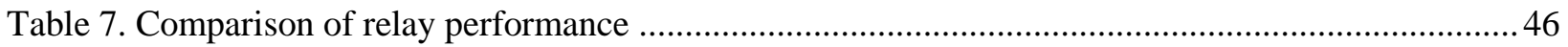




\section{$\underline{\text { List of Figures }}$}

Figure 1. Example of socket connection between Matlab and STK .................................................. 8

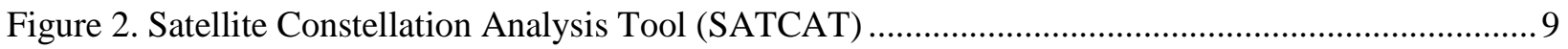

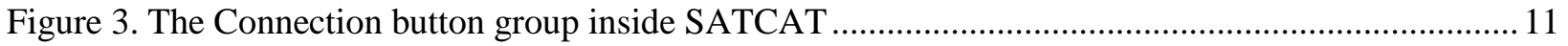

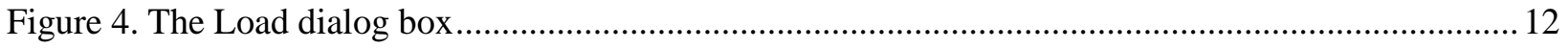

Figure 5. The scenario button group inside SACAT ............................................................................ 13

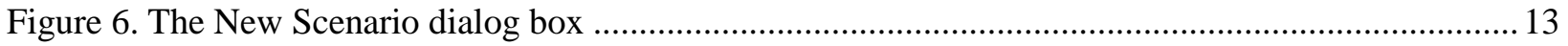

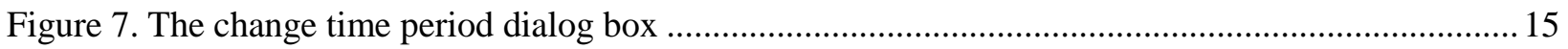

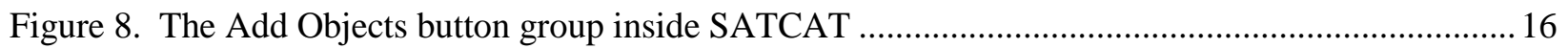

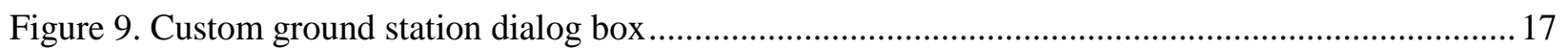

Figure 10. The Constraints button inside the Add Objects button group................................................. 18

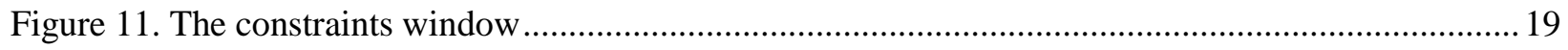

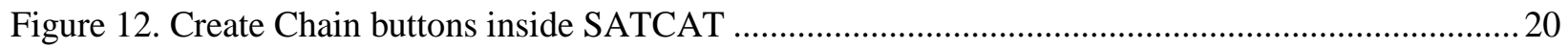

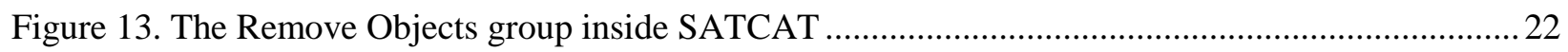

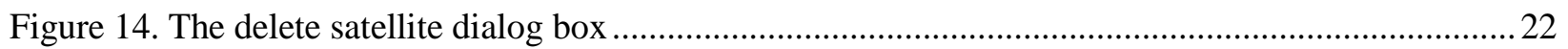

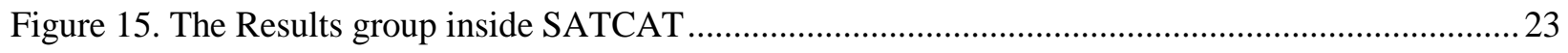

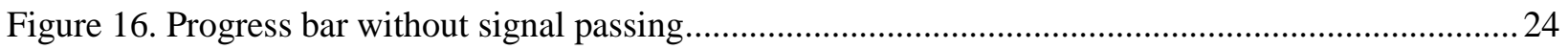

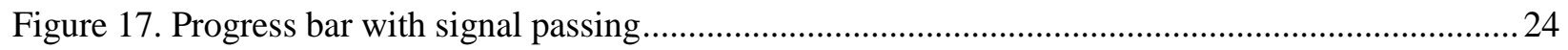

Figure 18. The plot section and drop-down menu inside SATCAT …..................................................25

Figure 19. The Create Ground Access Report button inside SATCAT ................................................26

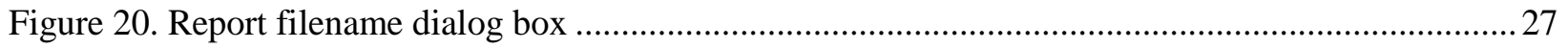

Figure 21. A sample TOA report between the Target and CubeSat constellation ....................................28

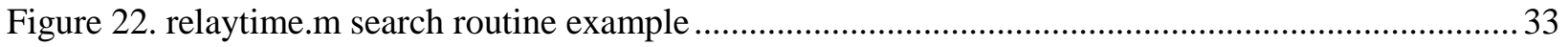

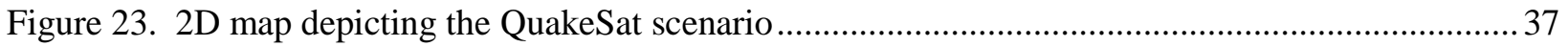

Figure 24. 2D map depicting the QuakeSat + 2 relay CubeSats scenario................................................ 37 
Figure 25. 2D map depicting the QuakeSat +36 CubeSat relay scenario

Figure 26. Relay time histogram for QuakeSat scenario

Figure 27. Relay time histogram for QuakeSat + 2 Relays.

Figure 28. (Left to Right) The number of acquisitions, passes, and deliveries per CubeSat.

Figure 29. QuakeSat + Constellation relay time histogram

Figure 30. (Left to Right) Acquisition, delivery and pass counts for QuakeSat + Constellation. 44

Figure 31. (Left to Right) Acquisition, delivery and pass counts for each CubeSat. .45 


\section{Background}

\section{$\underline{\text { CubeSats }}$}

The CubeSat project is an international collaboration of over 40 universities, high schools and private firms to design, test and build picosatellites containing scientific, private and government payloads. A CubeSat is a $10 \mathrm{~cm}$ cube with a mass of up to $1.33 \mathrm{~kg}$ that must conform to the CubeSat Standard. The CubeSat Standard is a joint partnership between California Polytechnic State University - San Luis Obispo (Cal Poly) and Stanford University to provide developers the necessary guidelines to build a CubeSat. The standard describes the outer dimensions, recommends materials, highlights restrictions and describes schedules pertaining to integration and launch. ${ }^{1}$ By creating the standard, it has simplified satellite infrastructure and allowed high schools and universities to design and produce a workable satellite at low cost. A basic CubeSat is often called a ' $1 \mathrm{U}$ ', for one unit, CubeSat and is 10x10x10 cm. The CubeSat Standard allows for scaling in one axis, by $1 \mathrm{U}$ increments allowing the production of ' $2 \mathrm{U}$ ' (20x10x10 cm) and '3U' (30x10x10 cm) CubeSats. Since all CubeSats are 10x10 cm, regardless of length, they can all be launched and deployed using a common deployment system. This system was designed by Cal Poly and is called the Poly-PicoSatellite Orbital Deployer (P-POD) which is mounted to a launch vehicle and can deploy $1 \mathrm{U}, 2 \mathrm{U}$ or $3 \mathrm{U}$ CubeSats in any combination up to a maximum volume of $3 \mathrm{U}^{2}$

\section{Current Applications}

Currently, over 70 CubeSats have been designed from different universities, private firms and government agencies. There are many different applications currently using CubeSats such as Earth remote sensing, biology, and space tether technology. Some examples of using CubeSats for Earth remote sensing include QuakeSat and SwissCube. QuakeSat, developed by 
Stanford University, was set out on a mission to help scientists improve earthquake detection by measuring changes in magnetic signals and SwissCube, built by one of the Swiss Federal Institutes of Technology, is conducting experiments with the air glow phenomenon in the Earth's atmosphere.

With the size and weight restrictions laid out in the CubeSat Standard, many developers are trying to integrate and test new technologies to improve the capabilities of CubeSats. Therefore, the main application for CubeSats currently is the development of new CubeSat technologies. PW-Sat, from the Warsaw University of Technology, is researching the use of an atmospheric drag device as a method to de-orbit CubeSats. AAU CubeSat, built by Aalborg University, was outfitted with a camera and a magnetically-based attitude control system to stabilize the CubeSat. With each successful test of new technology, CubeSats become more useful and capable of providing a quick, inexpensive platform for space payloads.

\section{$\underline{\text { Current Limitations }}$}

While research has gone into making CubeSats a viable space platform, one of the major limitations that CubeSats face is their access to ground stations in order to receive commands and downlink mission data. While there is a network of CubeSat ground stations, it takes time to transfer the data from one ground station to another in the event the owner's CubeSat doesn't pass over their own ground station. Also, if a command needs to be sent to the CubeSat, the ground must wait for it to pass overhead in order to uplink the commands. While this has worked in the past, the growing use of CubeSats for scientific payloads has increased and the amount of data each CubeSat can acquire is limited to onboard storage. With the help of a constellation, each CubeSat would be able to downlink data at a much faster rate than ever before, as well as receive commands from the ground even when the CubeSat is not directly overhead. Another approach to solve this problem would be to increase the number of ground 
stations available to the CubeSat. While this would allow for an increase in communication time between a CubeSat and ground stations, the cost associated with multiple ground stations is much higher than using a constellation of CubeSats. The cost to operate a ground station greatly exceeds the cost of adding CubeSats to a constellation. Additionally, it may not be possible to place ground stations worldwide in order to improve communication time to a point that is beneficial. 


\section{$\underline{\text { System Tool Kit }}$}

\section{$\underline{\text { Introduction }}$}

System Tool Kit (STK) is a modular physics-based software package developed by Analytical Graphics, Inc. ${ }^{3}$ that allows the complex analysis of land, sea, air and space assets. At the core of STK's software is a geometry engine that is designed to model time and space with unmatched accuracy that has been enhanced through more than 20 years of development and built to meet the needs of its users. STK delivers flexibility in coordinate system definition by converting from or to any coordinate system with equal accuracy. This flexibility allows users to define objects in the most appropriate coordinate system while maintaining a relationship with other objects. STK also determines dynamic spatial relationships among all of the assets, or objects, under consideration including the quality of those relationships or accesses given a number of complex, simultaneous constraining conditions. Additional flexibility on modeling the position, velocity and orientation of moving objects is provided to the user by allowing analysis using simple algorithms and fundamental equations to emulate ideal motion, or using high fidelity integrators and force models to generate realistic trajectories. ${ }^{4}$

STK was first adopted by the aerospace community for orbit analysis and access calculations (when one object can see another object). As the software was expanded, more modules were added that included the ability to perform calculations for communications systems, radar, interplanetary missions and orbit collision avoidance. In addition to the analysis provided, STK provides real-time visualization, in both 2D and 3D, of all air, land, sea and space components. This has allowed STK to become a standalone software package capable of modeling and analyzing complete and complex systems. Each system in STK is modeled with a hierarchy of objects, with the highest level object being a Scenario. A scenario will contain numerous parent objects and each parent object may include numerous child objects. 
In order to analyze the performance of a constellation of CubeSats, STK will be used to perform all orbital trajectory calculations, including perturbing forces. STK will also compute all access data between targets, satellites and ground stations, which will include access start and stop times, duration of access, and positional data for each access, such as azimuth, elevation and range while taking into account terrain and access constraints.

\section{$\underline{\text { Scenarios }}$}

Each analysis or design space within STK is called a scenario. Within each scenario and number of satellites, aircraft, targets, ships, communications systems or other objects can be created. Each scenario defines the default analysis time period and base units which are passed to all parent and child objects included in the scenario. These properties can be overridden for each object individually, as needed, with STK accurately making any conversions necessary for analysis. Only one scenario may exist at any given time; however, data may be exported and reused in subsequent analyses.

\section{$\underline{\text { Objects }}$}

Objects are at the base of STK's analysis engine. In order for a scenario to be meaningful, more than one object needs to be included. Once more than one object is added, STK can analyze relationships between two or more objects. These relationships can include: line of sight, position, velocity, communication duration or range to name a few. There are several different types of objects that can be added, such as: satellites, aircraft, ground vehicles, ships, targets, facilities, planets, stars, constellations or chains. Each object automatically inherits the general properties of the scenario, such as analysis time and base units. The properties of each object can be individually modified to fit the needs of the system being modeled, allowing for different coordinate systems, units or analysis time periods for each object. 
The objects that will be utilized to analyze the performance of a constellation of CubeSats include: one target, multiple satellites, multiple facilities (or ground stations), one constellation of satellites, one constellation of ground stations, and three chains; one linking the target to the satellite constellation, one linking the satellite constellation to itself and one linking the satellite constellation to the ground station constellation. STK treats a target object and facility object in exactly the same way. The only difference between the two is the icon used in the visualization portion of STK. A constellation object allows other objects to access any member of the constellation.

\section{$\underline{\text { Reports }}$}

STK analyzes the complex relationships among multiple objects, regardless of the data's origin. By modeling line of sight visibility or by adding constraints like terrain, communication parameters or sensor pointing, users can realistically determine when objects can interact with each other. In order to understand how a system is performing the user must have access to the relationship data between objects that STK provides. For each object within a scenario, various reports and graphics, both static and dynamic, may be created. Relative parameters between one object and another can also be reported and the effect of real-world restrictions, or constraints, enabled so that more accurate reporting is obtained. Through the use of constellations and chain objects, multiple objects may be grouped together and multi-path interactions between them may be investigated.

While STK has the ability to analyze many complex systems and provide dynamic spatial relationship data between multiple objects, it is not capable of analyzing a communication system based on a store-and-forward model. The current functionality of STK only allows for the analysis of an instantaneous communication chain, i.e. when all objects in the chain are visible to all other objects in the chain simultaneously. A store-and-forward model requires 
knowledge of which objects have stored the data at which time, and when that object is able to forward the data to another object. In order to accomplish this task, a modular external function is required expand the capabilities of the STK Engine. Utilizing access reports provided by STK, an algorithm will be developed to analyze the performance of a CubeSat constellation. The report that will be utilized in analyzing constellation performance is a custom Time Ordered Access (TOA) report. A TOA report lists all available accesses between two objects in chronological order, starting with the earliest and ending with the latest access. The information provided by this report includes: accessed object names, access start and end times, access duration and azimuth, elevation and range between the two objects. Three TOA reports are required; one between a target and the satellite constellation, one between the satellite constellation and itself and one between the satellite constellation and the ground station constellation. With these three reports, the relay performance of the constellation can be determined.

\section{$\underline{\text { External Control }}$}

An added benefit of STK is the ability to control it through an external application through TCP/IP or Component Object Model (COM). The COM library contains types, interfaces, events and classes representing various aspects of the STK application structure, which is designed to make it easy for a user to build custom solutions using STK and the STK Engine. The various components provide the user with the capability to control and automate STK objects, manage the lifetime of STK objects, access Data Provider Tools, perform access and coverage computations and respond to events generated by STK. ${ }^{5}$ Both integration techniques make use of the connect scripting language to accomplish the task. Connect is a messaging format that is completely language independent. This allows applications and client 
tools to be created in the user's programming language of choice. A socket connection, as shown in Figure 1, allows for commands and information to be sent between Matlab and STK.

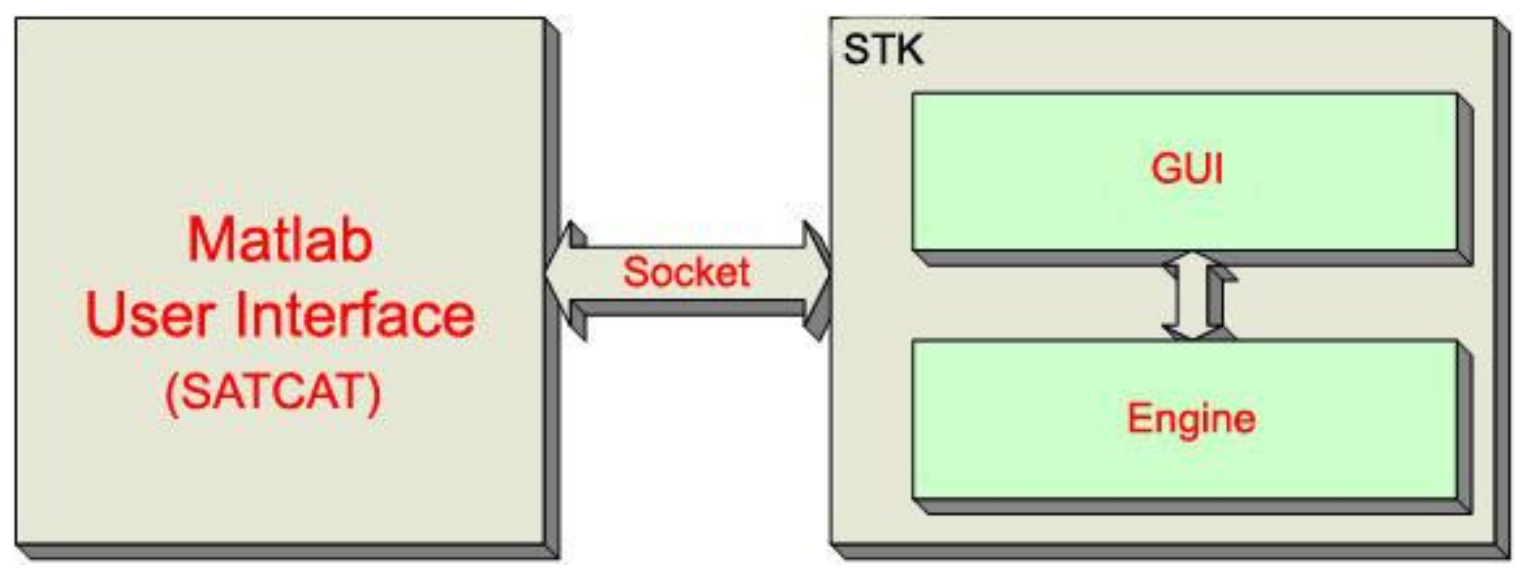

Figure 1. Example of socket connection between Matlab and STK

The advantages of using an external application to control STK include the ability to automate the generation and properties of STK objects to meet a design requirement or to analyze a model not currently supported by the STK Engine. Since the STK Engine does not support communication links based on a store-and-forward model an external application is needed to provide this functionality. Using Mathwork's Matlab ${ }^{6}$, a graphical user interface (GUI) will be created to provide the user with the ability to control STK externally and analyze a constellation based on a store-and-forward model. 


\section{Satellite Constellation Analysis Tool}

\section{SATCAT Introduction}

The Satellite Constellation Analysis Tool (SATCAT) is a Matlab GUI, shown in Figure 2, which has the ability to send commands to and receive information from STK. By controlling STK externally, user created functions can be utilized to compute data, or analyze models that are not currently supported inside of the STK Engine. SATCAT was designed to be a modular external interface with STK, allowing various custom user created functions to be called to perform analysis or design optimization.

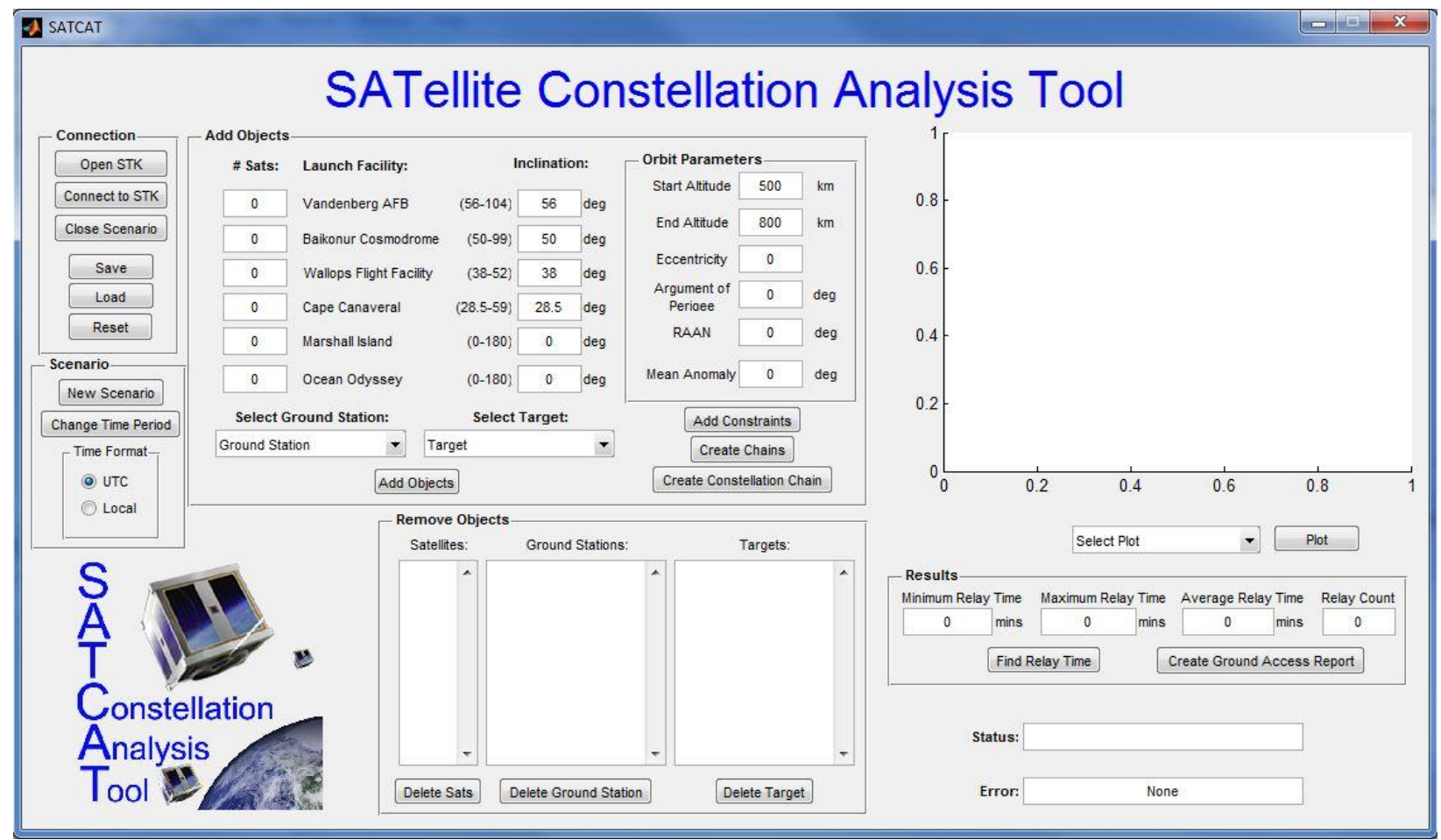

Figure 2. Satellite Constellation Analysis Tool (SATCAT)

In its current configuration, SATCAT is capable of establishing a socket connection with STK, creating a new scenario and defining the scenario analysis time period, adding and removing CubeSats, ground stations and targets, creating communication chains, calculating the relay performance of a defined scenario, plotting statistical relay performance data, creating a 
ground station access report, changing the analysis time period, and saving and loading scenarios. Each of these features will be discussed in the following sections. The modular nature of SATCAT allows the user to create new or remove functionality depending on their specific needs.

Connect commands are the language independent method for controlling STK externally. By sending a Connect command through the open socket connection, the user is able to control every aspect of the STK user interface and STK Engine externally. After STK has completed the command, it returns verification that the task has been completed to Matlab.

\section{$\underline{\text { Connection }}$}

The button group "Connection", shown in Figure 3, contains buttons for opening an instance of STK, creating the socket connection between Matlab and STK, closing a current scenario, saving, loading and resetting SATCAT. In order for a socket connection to be made between Matlab and STK, both programs need to be open and running on the user's computer. If STK is not currently running, the user has the ability to open STK directly from SATCAT using the "Open STK" button. Once both programs are running, a socket connection can be established using the "Connect to STK" button. When the user clicks the "Connect to STK" button, the Matlab/STK interface function, stkInit, is called which opens a port on the user's machine allowing communication between Matlab and STK. 


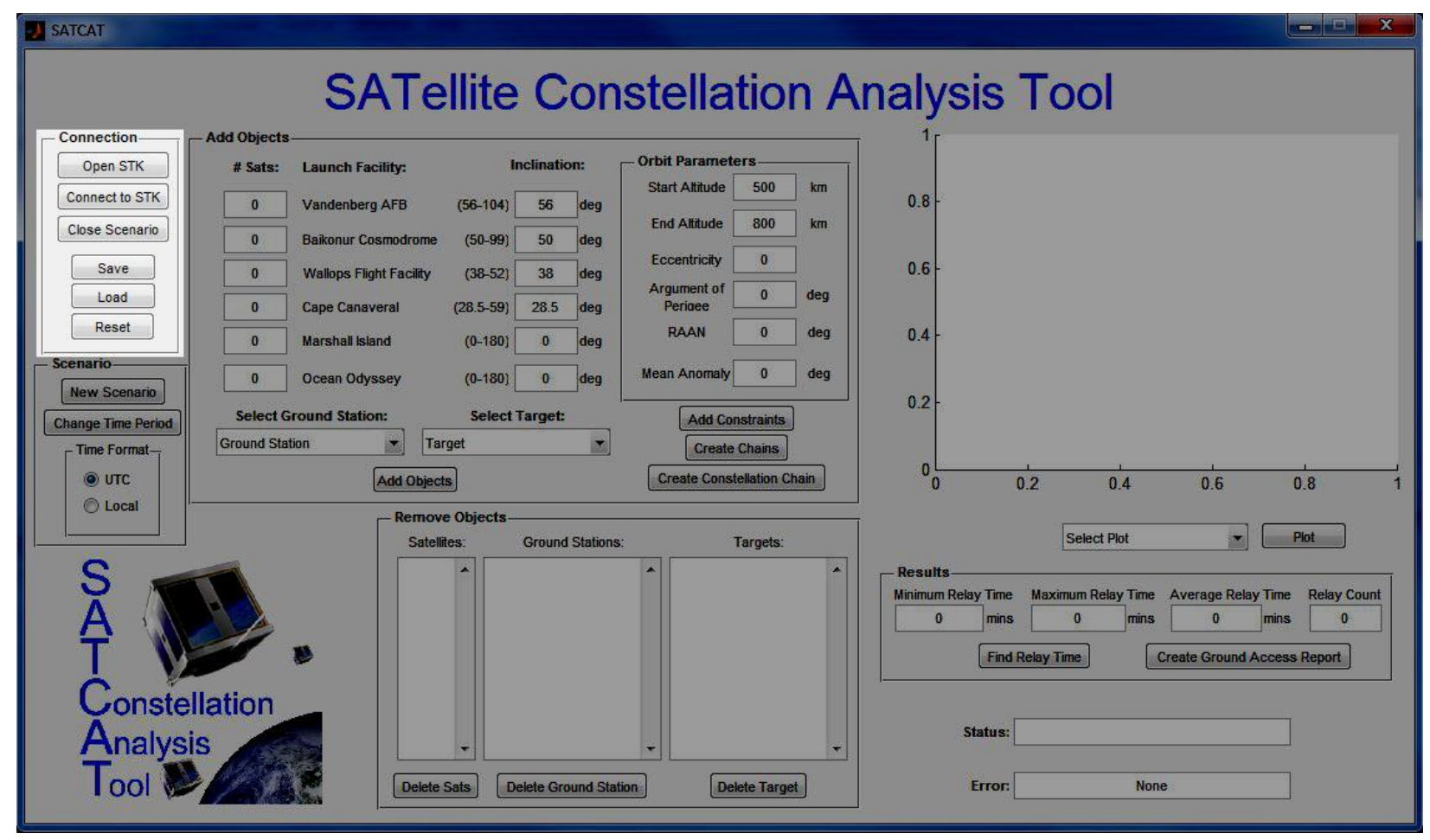

Figure 3. The Connection button group inside SATCAT

After a scenario has been created and populated with objects, the scenario may be saved for future use by clicking the "Save" button. Clicking "Save" automatically creates a new folder and saves the scenario with a filename that matches the user defined name of the scenario. If a user wishes to load a previously saved scenario, clicking the "Load" button brings up the Load dialog box, as shown in Figure 4. The user must then enter the name of the scenario they wish to load. If the user incorrectly types, or requests an invalid scenario, a warning will alert the user that no valid scenario was found to load. When a user loads a scenario, the original analysis time period is kept and all objects that are associated with that scenario are loaded into SATCAT. STK will also propagate all objects to the analysis time period once the scenario is loaded. Once the scenario has successfully loaded, and STK has finished propagating, objects may be added or removed and the performance of the constellation analyzed. 


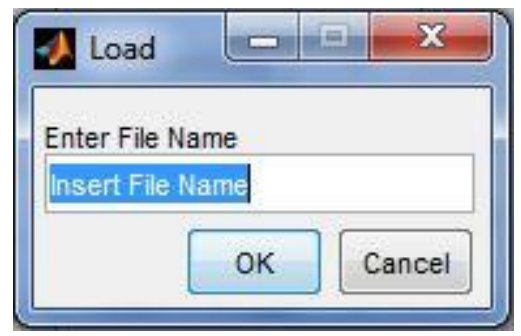

Figure 4. The Load dialog box

If the user wishes to close the current scenario in order to load or create a new scenario, clicking the "Close Scenario" button closes the current scenario in STK and resets all variables in SATCAT. If, for some reason, a complete reset of SATCAT and the connection to STK is required, clicking the "Reset" button will clear and reset all variables in SATCAT and close the socket connection between Matlab and STK. Following a reset, a new connection must be established between Matlab and STK by clicking the "Connect to STK" button.

\section{$\underline{\text { Scenario }}$}

Once a connection has been made between Matlab and STK a scenario must be created. The user has the option to either load a previously saved scenario, or create a new scenario. The "Scenario" button group, shown in Figure 5, allows the user to create a new scenario, change the analysis time period of the current scenario and choose the time format for the scenario and any reports generated. 


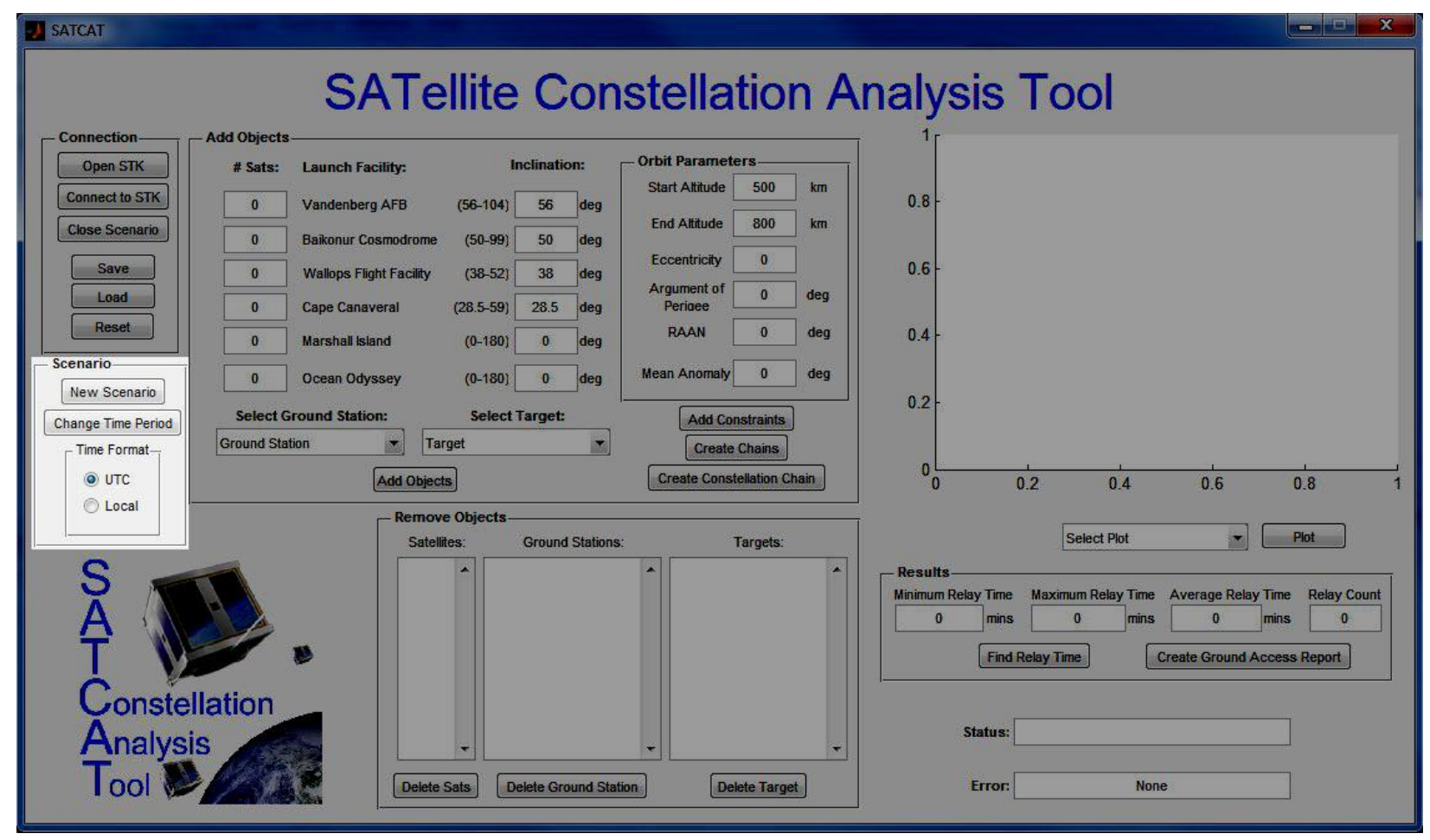

Figure 5. The scenario button group inside SACAT

When the "New Scenario" button is clicked a dialog box will pop-up, shown in Figure 6,

allowing the user to enter the scenario name, start time and end time.

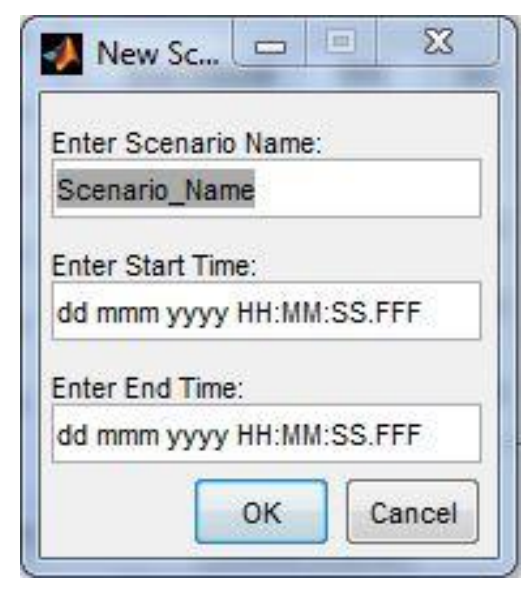

Figure 6. The New Scenario dialog box

Every scenario requires the user to define the times during which the conditions that are set for the scenario, and the objects in the scenario, will be relevant. The analysis time period defines the epoch and the start and end times of the scenario. The "SetAnalysisTimePeriod" Connect 
command is used to establish the analysis time period of the scenario. The user has several options on how to define the start and end times. The first is simply entering a date and time in the form dd mmm yyyy HH:MM:SS.FFF; where dd is the day, mmm is the three letter month, yyyy is the year, and HH:MM:SS.FFF are hours, minutes, seconds and milliseconds. The stop time can also be entered as an increment from the start time in the form " $+\mathrm{n}\{$ Unit $\}$ ", where $\mathrm{n}$ is an integer and $\{$ Unit $\}$ can be any of the following: Day, Days, Hour, Hours, Hr, Hrs, Min, Mins, Minute, Minutes, Sec, Secs, Second, Seconds. Other options for start time are shown in Table 1.

Table 1. Valid start time inputs

\begin{tabular}{|cc|}
\hline Input & Start Time \\
\hline "Now" & Current date and time of user's location \\
\hline "Today" & Current local date at either 12:00:00 local time or 00:00:00 UTC \\
\hline "Tomorrow" & Tomorrow's date at either 12:00:00 local time or 00:00:00 UTC \\
\hline
\end{tabular}

Once the user enters the scenario name and start and end times, STK will create a new scenario with the user's inputs. In addition to creating the new scenario in STK, several objects are added by default. These objects include a Satellite Constellation, a Ground Station Constellation, a Target-to-Constellation chain, a Constellation-to-Constellation chain, and a Constellation-to-Ground Station Constellation chain. As CubeSats and ground stations are added, they will automatically be placed into their corresponding constellation object. These objects are required to analyze a data relaying CubeSat constellation based on a store-andforward model. Before any analysis is done, the user has the option to change the time format of any reports created within STK. Analysis can be done in either Universal Time (UT) or Local time and can be changed at any point before reports are generated.

If it is necessary to determine the performance of a given scenario at some time in the future, the user has the ability to change the analysis time period by clicking the "Change Time Period" button. Once clicked a dialog box will pop-up, shown in Figure 7, allowing the user to 
define a new analysis start and end time. Valid inputs for the start and end time are the same as when creating a new scenario.

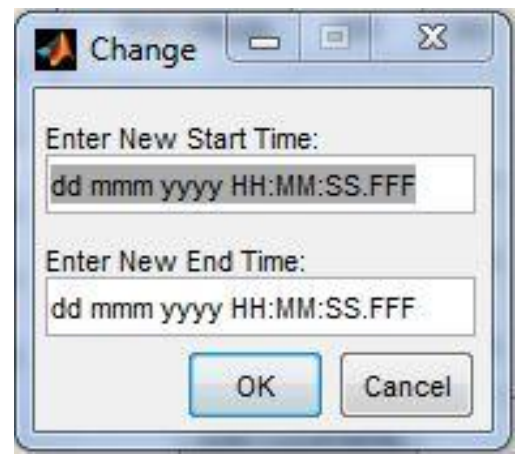

Figure 7. The change time period dialog box

When changing the analysis time period, STK will re-propagate all objects to the new analysis time period. Depending on how many objects are currently in the scenario and how far into the future the new analysis time is, it may take a considerable about of time for STK to compute the new CubeSat ephemeris data.

\section{$\underline{\text { Add Objects }}$}

In order to create a meaningful scenario, objects must be added so as to investigate their spatial relationships. The "Add Objects" button group, shown in Figure 8, allows the user to add satellites and define their specific orbit, add ground stations, a target, create the required communication chains and place constraints on the CubeSat constellation, ground stations or target. 


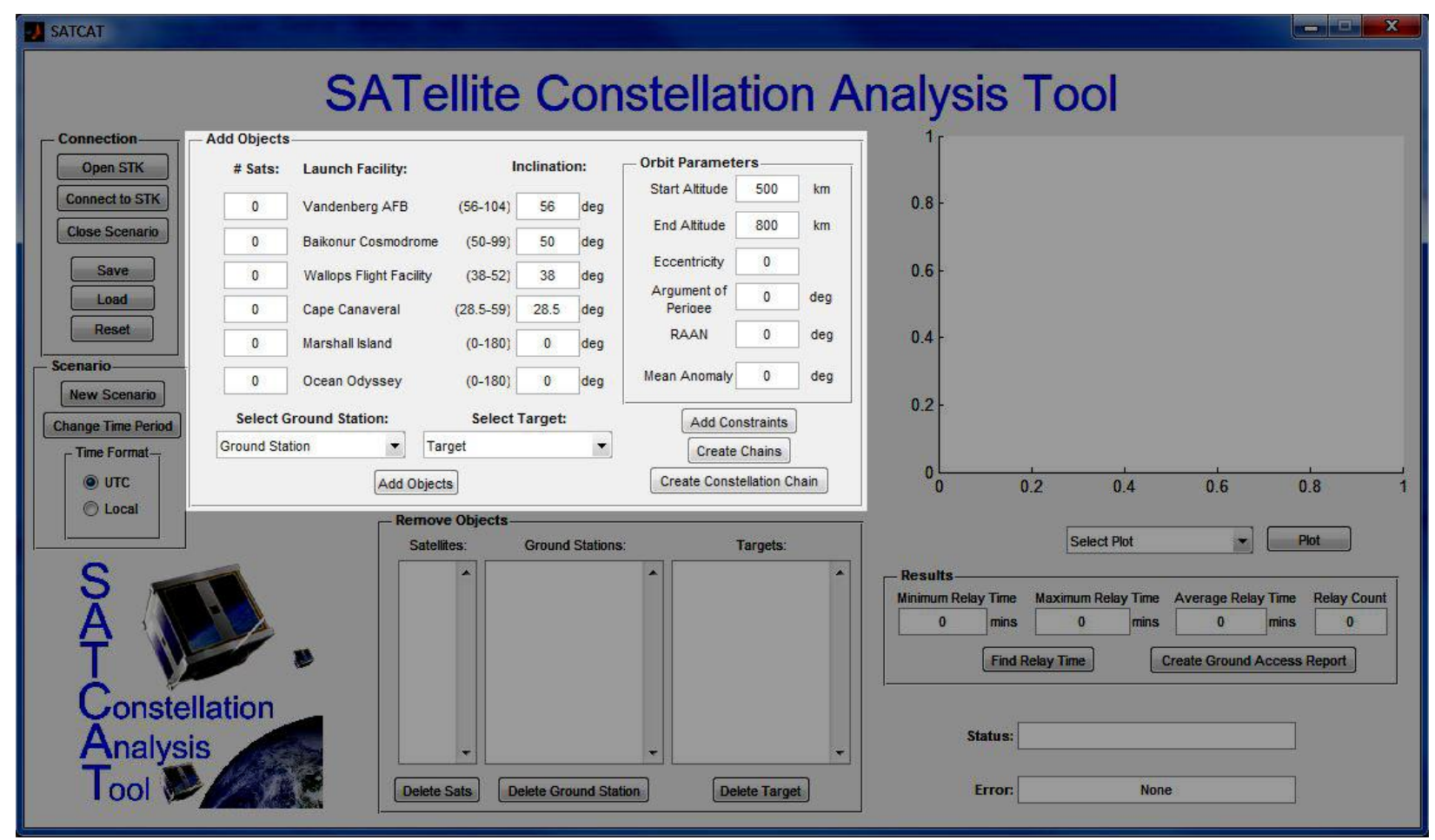

Figure 8. The Add Objects button group inside SATCAT

Any number of satellites may be added to the scenario simply by entering the number of satellites to be added next to the desired launch site. Each launch site is capable of launching payloads into particular inclinations denoted under the heading "Inclination". In the "Orbit Parameters" section, the user has the ability to define the orbits exact size, shape and orientation as well as the initial position of the CubeSat. If more than one satellite is being launched from a single launch site, then the "Start Altitude" and "End Altitude" determine the altitude spacing between each satellite. Satellites will be evenly spaced between the "Start Altitude" and "End Altitude", with the first satellite being placed at the "Start Altitude", i.e. if three CubeSats were added from Cape Canaveral with a Start and End Altitude of $500 \mathrm{~km}$ and $800 \mathrm{~km}$, respectively, then CubeSats would be placed at $500 \mathrm{~km}, 650 \mathrm{~km}$ and $800 \mathrm{~km}$. This allows for multiple CubeSats released from the same launch vehicle to be placed into slightly higher or lower orbits. If a pseudorandom orbit is desired, entering "rand" into the "Argument of Perigee", "RAAN" or "Mean Anomaly" field will generate random values for those orbital elements. 
A list of major U.S. cities is provided in the ground station drop-down menu, and major world cities in the target drop-down menu for the user to select. The option to add a custom ground station, shown in Figure 9, or custom target is provided in each drop-down menu if the user wishes to define their own location. The name, latitude (North) and longitude (East) is required for each custom location added.

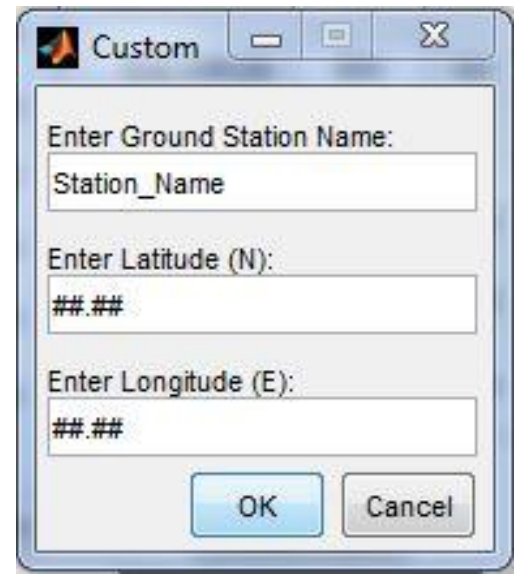

Figure 9. Custom ground station dialog box

Once the objects to be added have been selected, clicking the "Add Objects" button sends the command to STK to add the desired objects to the scenario and propagate all satellites to the current analysis time period. At the same time, every satellite gets added to the Satellite Constellation and the selected ground station gets added to the Ground Station Constellation. The "Add Objects" button may be used multiple times in order to add all desired satellites and ground stations.

\section{$\underline{\text { Add Constraints }}$}

Once all the desired objects have been added, constraints may be placed on those objects to model a more accurate system. Clicking the "Add Constraints" button, seen in Figure 10, brings up the constraints window. Included in the constraints window, shown in Figure 11, is the 
ability to set lighting constraints and temporal constraints on satellites, ground stations and the target.

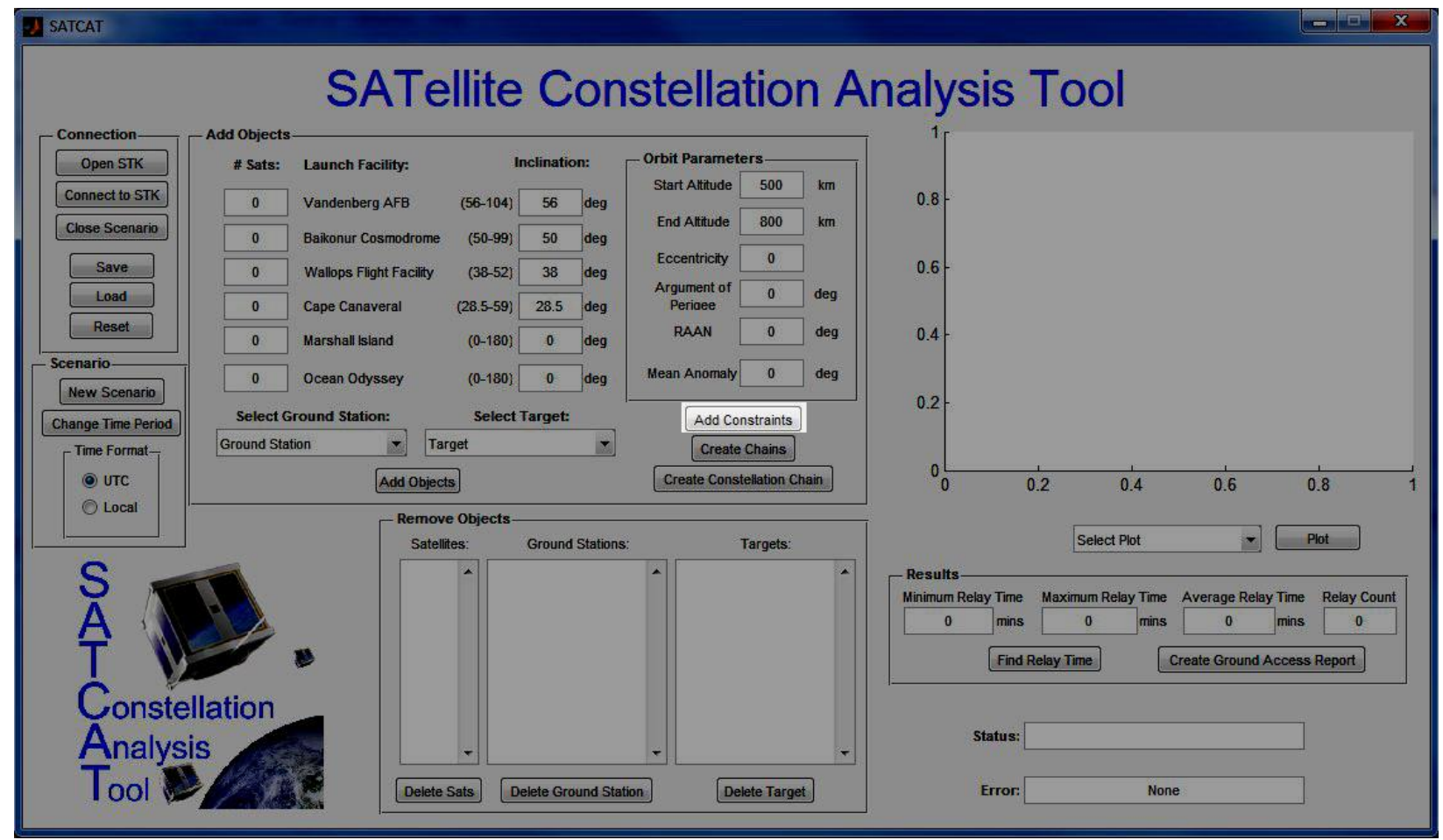

Figure 10. The Constraints button inside the Add Objects button group

Constraints allow limitations to be placed on the performance of objects. All constraints work in an And configuration meaning that when multiple constraints are set, they all must be satisfied in order for an access to occur. If any single constraint is violated, that access is invalidated.

Lighting constraints available include the ability to require an object to be in direct sun, penumbra or direct sun, penumbra or umbra, penumbra, umbra or direct sun, or umbra and can be placed on satellites, ground stations or the target. The azimuth angle, elevation angle and range in the "Satellite" section define the minimum or maximum position between satellites that must be met in order for access to occur. Likewise, the elevation angle in both the "Facility" and "Target" sections sets the minimum or maximum elevation that must be satisfied. Specific properties of the satellite are also available to be set in order to improve the accuracy of the 
orbital propagation. By setting the mass, area-to-mass ratio and coefficient of drag, STK can more accurately model the perturbing forces on the satellite. When propagating satellites, STK takes into account several different perturbing forces including drag, solar radiation pressure, earth oblateness and $3^{\text {rd }}$ body effects. STK uses the area-to-mass ratio in determining the perturbing forces due to both drag and solar radiation pressure. The user should be aware however, that currently there is no way to define areas for drag and solar radiation pressure separately, and as such, STK will use the area-to-mass ratio for both perturbing forces. The final constraint available is the ability to define how long it takes for a communication link to be established and the data transferred between objects and may be set in the "Acquisition Time" input box.

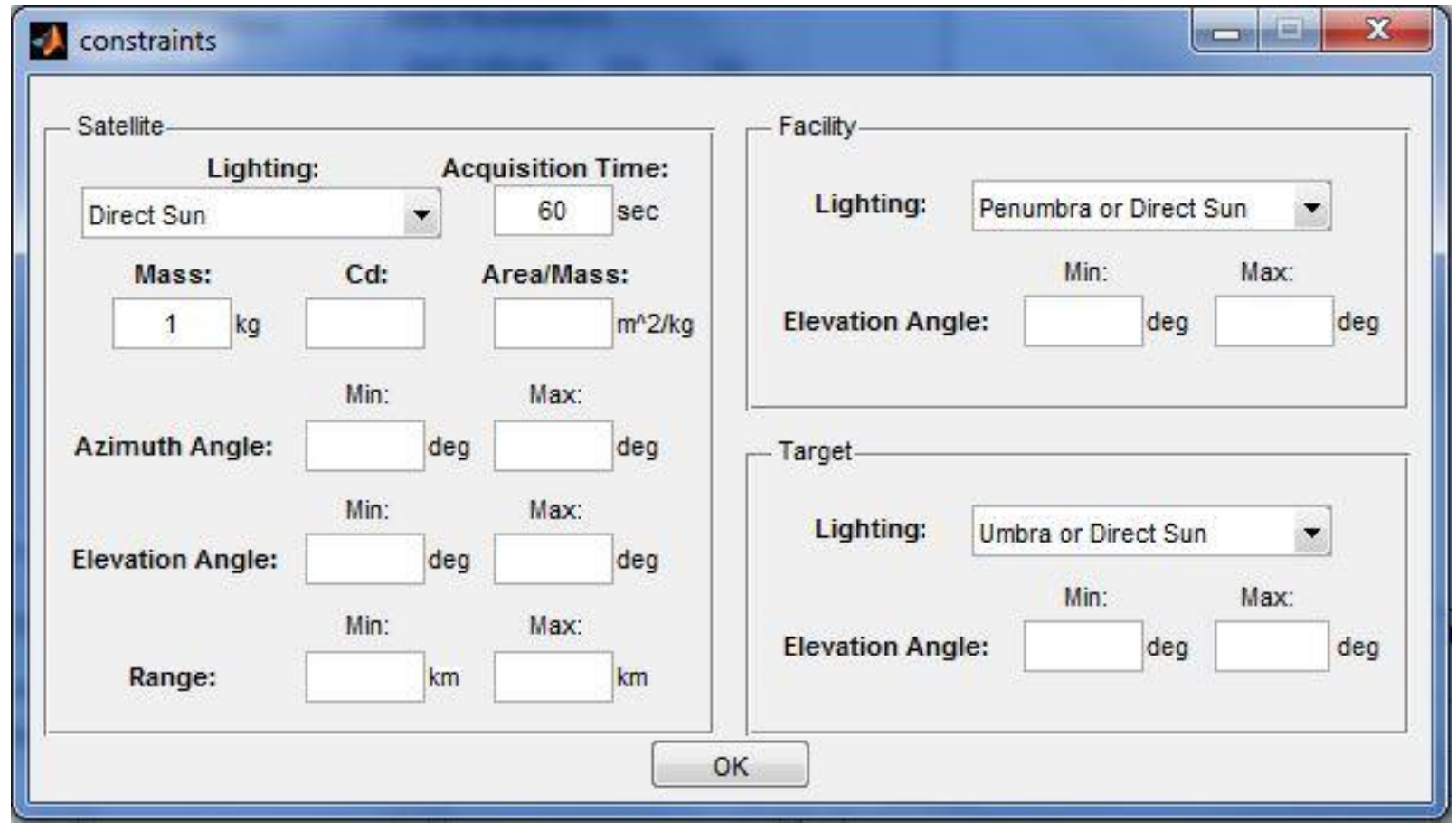

Figure 11. The constraints window

After defining the desired constraints, clicking the "OK" button will send the constraints to STK for each object. Constraints are only placed on currently added objects in the scenario 
when the "OK" button is clicked. Any objects added after setting constraints will not have constraints placed on them unless the user goes through the "Add Constraints" process again.

\section{Create Chains}

There are three different chains required to analyze the performance of the constellation. Depending on the type of scenario built there are two possible options for creating the communication chain. If a target has been added to the scenario, the user must click the "Create Chain" button, shown in Figure 12. Creating this type of communication chain allows every satellite in the constellation to receive data from the target, pass the data to any other satellite in the constellation and downlink the data to any ground station. The three chains required for this option include one between the ground target and the CubeSat constellation, one between the CubeSat constellation and itself and one between the CubeSat constellation and the ground station constellation.

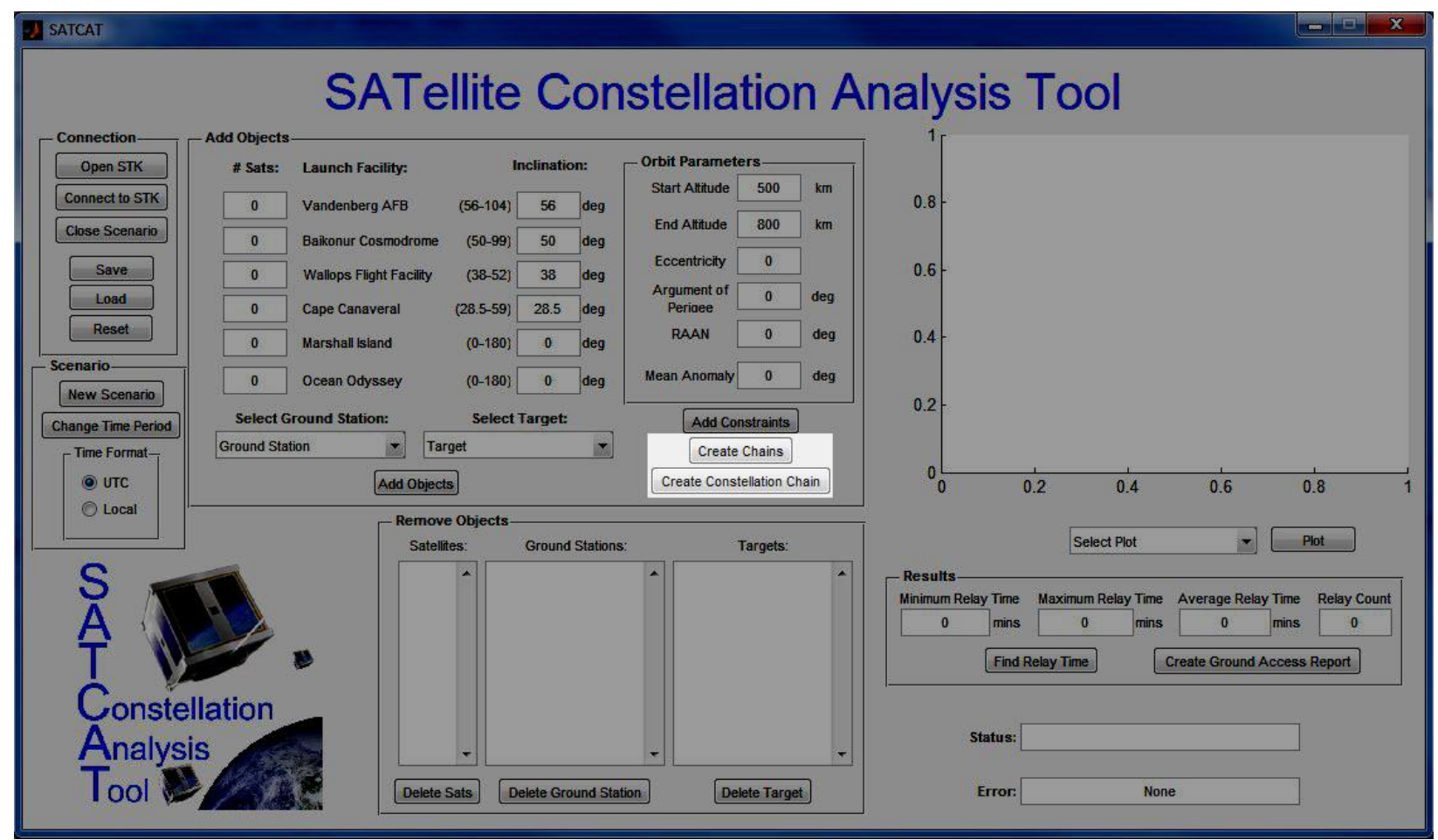

Figure 12. Create Chain buttons inside SATCAT 
If no target has been added then the user must click the "Create Constellation Chain" button, also shown in Figure 12. This communication chain sets the first CubeSat added as the target as well as part of the Satellite Constellation. This allows the analysis of a CubeSat relay constellation where an independent CubeSat can access the constellation in order to improve communication response. Instead of a user waiting for their individual CubeSat to pass over one of their ground stations in order to uplink commands or downlink data, the user has access to the entire constellation network; therefore reducing the communication latency and increasing the number of accesses. The three chains required for this option include one between the first CubeSat added to the scenario and the CubeSat constellation, one between the CubeSat constellation and itself and one between the CubeSat constellation and the ground station constellation.

\section{$\underline{\text { Remove Objects }}$}

In addition to the ability to add objects to a scenario, the user has the option to remove any CubeSat, ground station or target. A list of the currently added CubeSats, ground stations and target are displayed in the "Remove Objects" group, shown in Figure 13. 


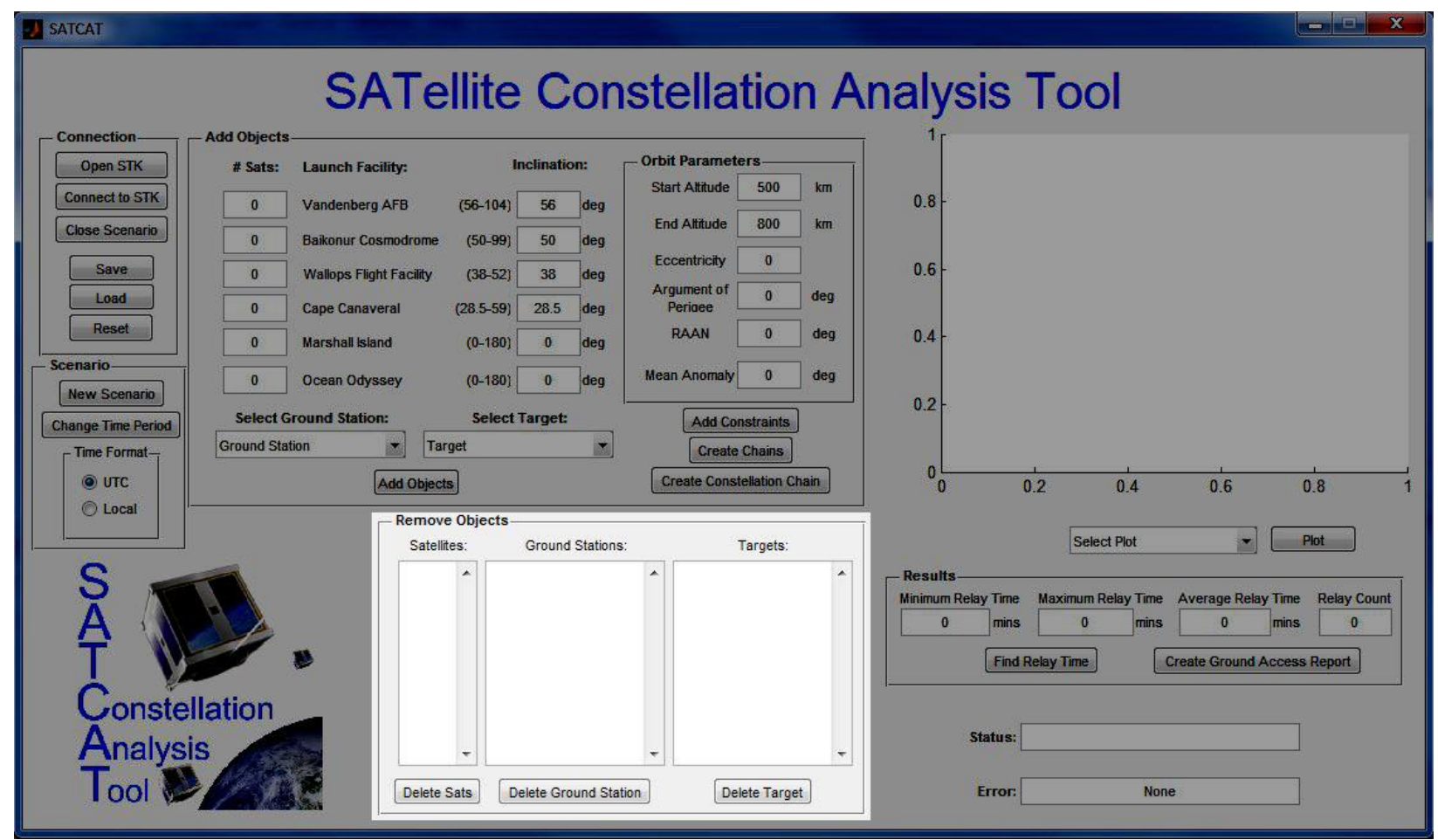

Figure 13. The Remove Objects group inside SATCAT

In order to remove an object, the user must click the delete button corresponding to the object to be removed. When the button is clicked a delete dialog box, shown in Figure 14, will appear requesting the name of the object to be removed. After entering the name, clicking the "OK" button will send the command to STK to remove the requested object.

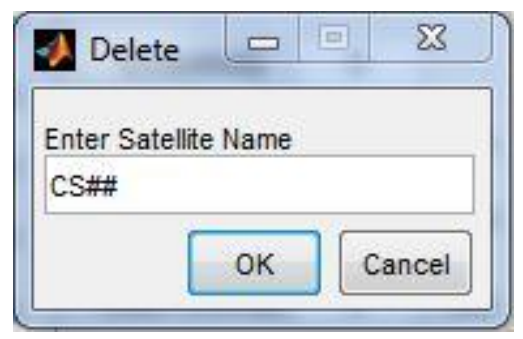

Figure 14. The delete satellite dialog box

If the user tries to remove an object that does not exist, or incorrectly types the name of the object, a warning will be displayed and the user must attempt to remove the object again.

Removing an object also removes it from any associated constellations or chains in the scenario. 


\section{$\underline{\text { Results }}$}

After the user has created a scenario, populated it with objects and set constraints on those objects, the constellation performance can be analyzed. Clicking the "Find Relay Time" button in the "Results" group, shown in Figure 15, requests and receives the TOA reports from STK and sends them to a custom designed search function, relaytime.m, to calculate the constellation performance.

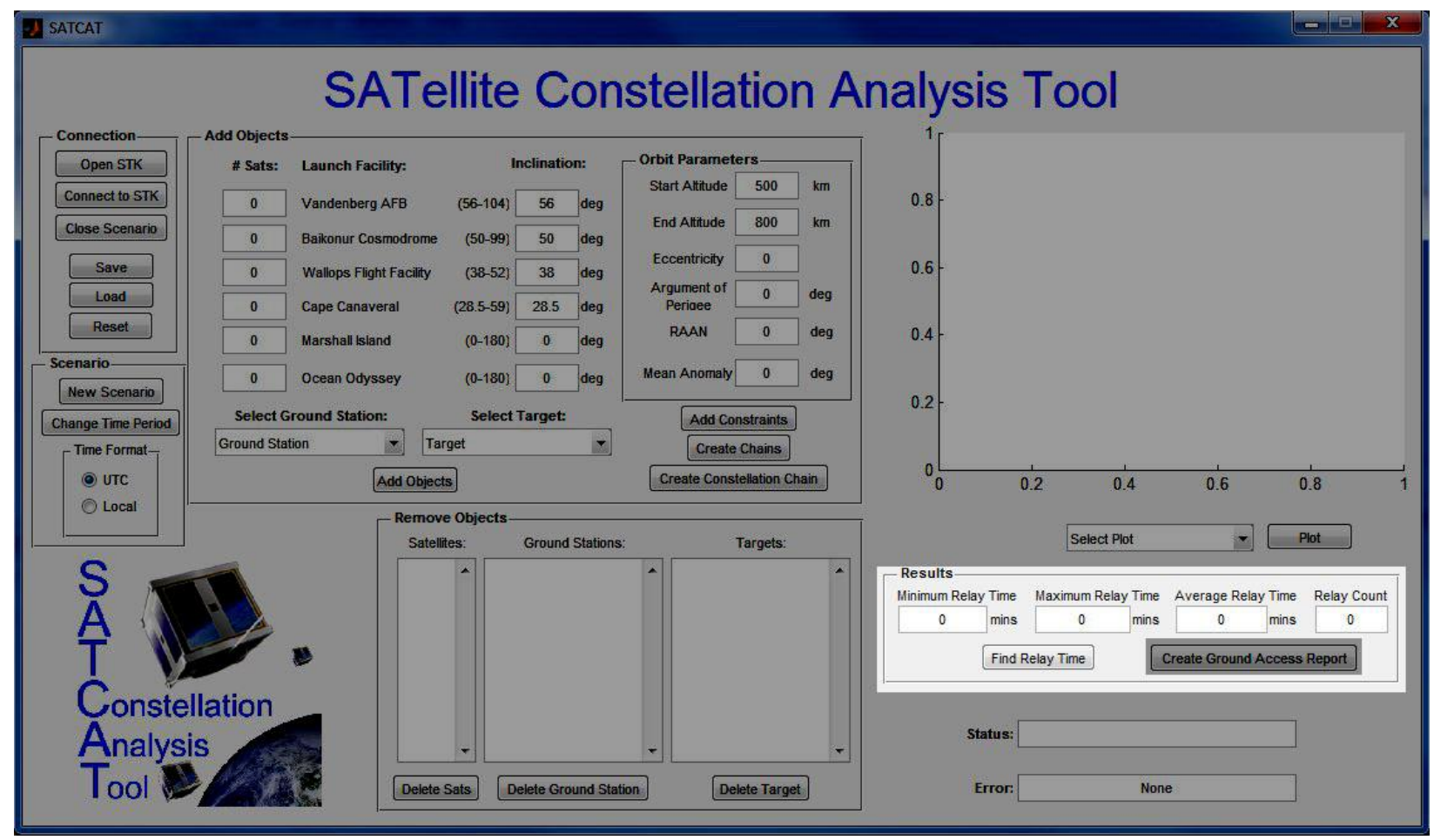

Figure 15. The Results group inside SATCAT

Calculating the constellation performance provides the user with the minimum, maximum and average time it takes to relay a signal from the target object, through the CubeSat constellation and to a ground station as well as how many successful relays were performed in the analysis time period. Two possible progress bars will show the progress of the CubeSat constellation performance calculation, shown in Figure 16 and Figure 17. If a data package is never relayed to another CubeSat, or if the scenario only contains one CubeSat, then the first progress bar will display the progress of a simple relay calculation. Otherwise, the second progress bar will 
appear and show the progress of the relay, and signal passing between CubeSats in the constellation. In either case, the computation is complete with the "Relay" bar reaches $100 \%$.

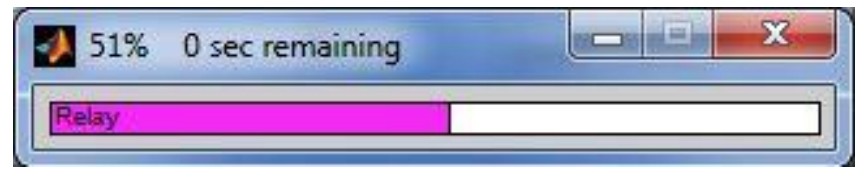

Figure 16. Progress bar without signal passing

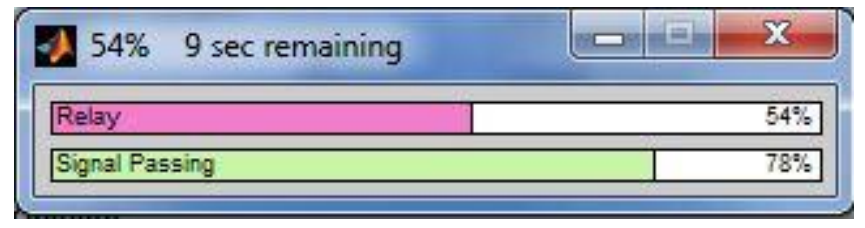

Figure 17. Progress bar with signal passing

Once complete, the results will be displayed in the corresponding output box in the "Results" group. If no relay is found for the given scenario "None" will be displayed in minimum, maximum, average and relay count output boxes. After the constellation performance results have been displayed, all objects are removed from the chains, requiring the user to click one of the "Create Chains" buttons again before re-calculating constellation performance.

\section{$\underline{\text { Plots }}$}

While minimum, maximum and average relay times are important in determining the performance of a constellation they do not show the whole picture. By visually analyzing the data, a user can have a better understanding of the system performance. Information like relay time distribution or how many times each satellite participated in the constellation can provide another perspective on performance. The plot section, shown in Figure 18, provides the ability to visualize the other performance characteristics of the constellation. 


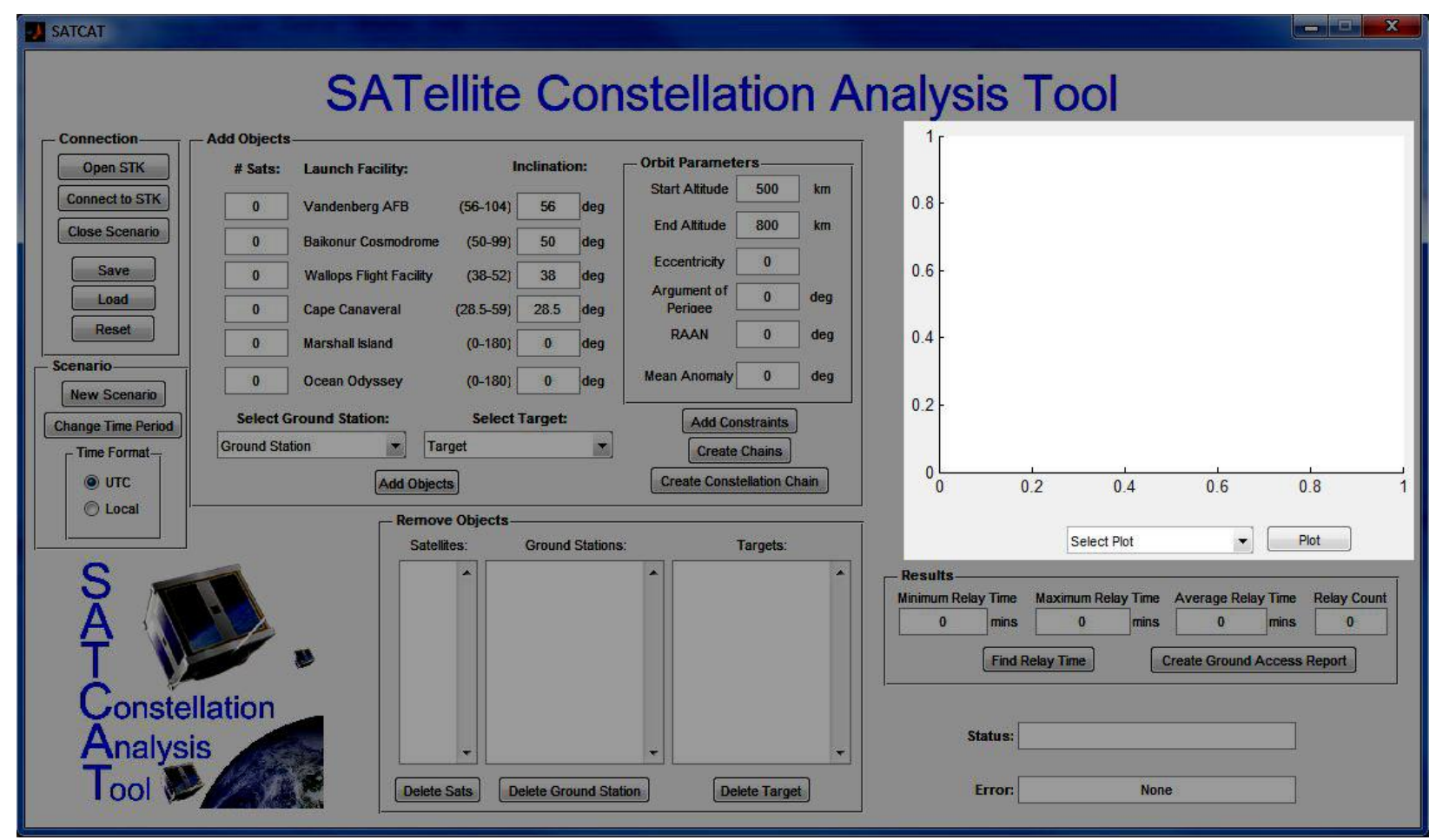

Figure 18. The plot section and drop-down menu inside SATCAT

There are four different plots available, including a relay time histogram, CubeSat data acquisition count, data pass count and data delivery count. The relay time histogram shows the distribution of relay times between the minimum and maximum relay time. This allows the user to visualize where most of the times are located, whether they are grouped closer to the minimum time, maximum time, or scattered randomly between the two. The other three plots display how many times each CubeSat acquired data from the target, passed the data to another CubeSat, and delivered the data to a ground station. This allows the user to analyze the performance of each CubeSat in the constellation. If one or more CubeSats are not contributing to the constellation, either by not acquiring, passing or delivering data, then the user has the ability to identify and remove them. In order to display each of these graphs, the user must select the desired plot from the drop-down menu, followed by clicking the "Plot" button. 


\section{Ground Access Report}

The "Create Ground Access Report" button, shown in Figure 19, serves two purposes.

The first is it allows the user to create an access report that details the time and location of every successful data delivery between the CubeSat constellation and the ground stations. Included in the report is the name of the ground station that receives the data, the start and end date and time and start and end azimuths, elevations and ranges between the ground station and CubeSat.

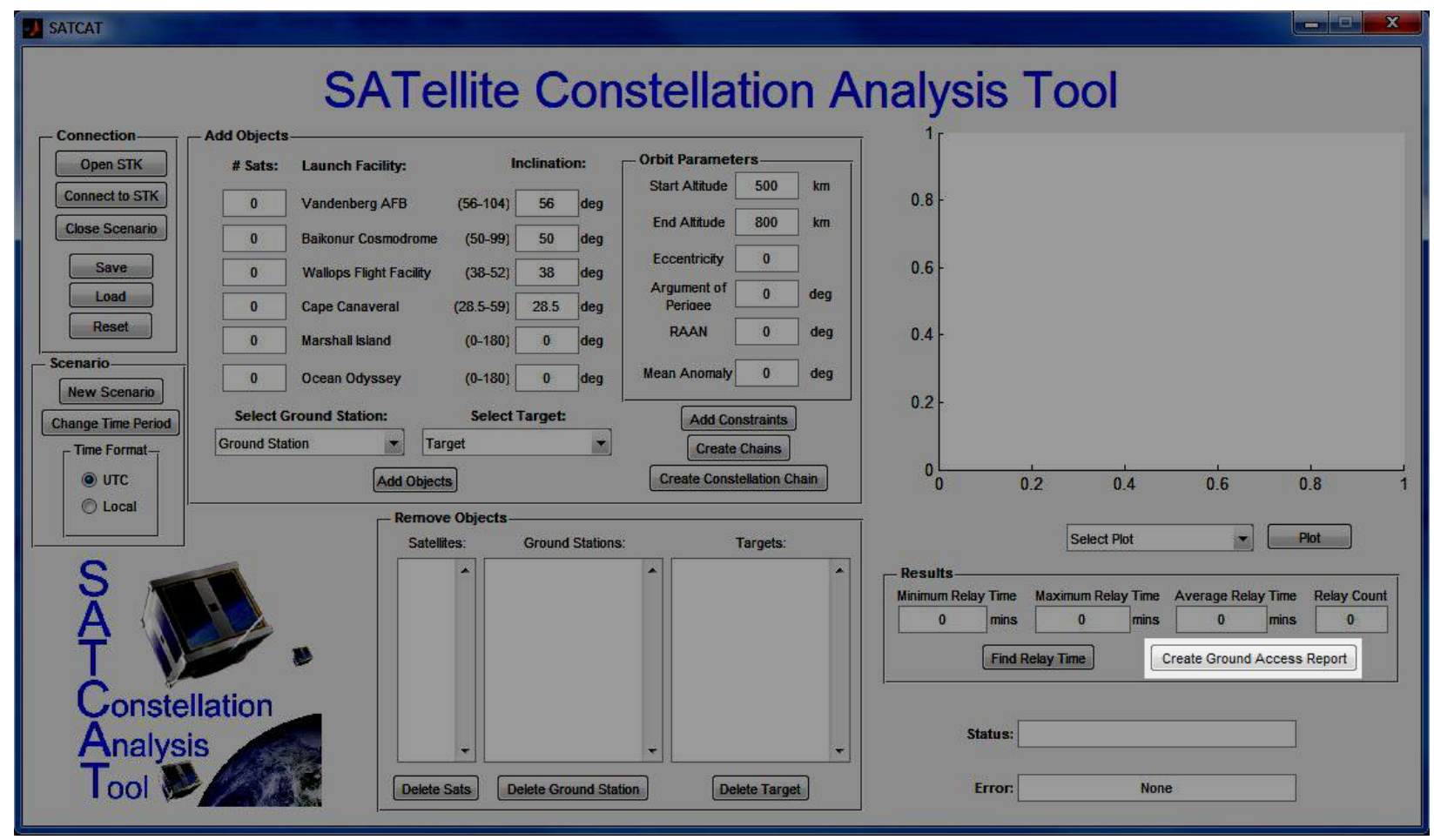

Figure 19. The Create Ground Access Report button inside SATCAT

This allows the ground station to track the correct CubeSat as it passes overhead in order to receive the data package. The second purpose of the report is that it also saves the constellation performance data so that it may be referenced or viewed at a later time without the need to recreate the scenario and re-compute the performance. At the bottom of each report the minimum, maximum and average relay times along with the number of successful relays is displayed and each plot is saved as a JPEG in the corresponding scenario folder. By saving the performance 
data and plots, a user can reference the information or compare two or more scenarios without re-creating each scenario.

When the user clicks the "Create Ground Access Report" button, a prompt, shown in Figure 20, requests a file name to be used to save the report and all plot JPEGs. Once the user enters a name the report data is sent to Excel and saved as an Excel spreadsheet and each graph is plotted and saved as a JPEG.

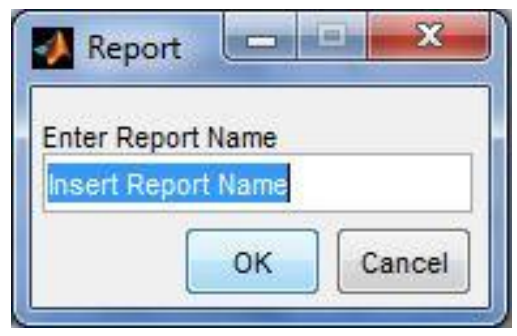

Figure 20. Report filename dialog box 


\section{$\underline{\text { Relaytime.m Function }}$}

\section{Introduction}

Currently, the STK Engine can only analyze a communication chain when that chain is complete at an instant in time. This only allows for instantaneous communication chains, where a store-and-forward model is a much more accurate representation of real world scenarios. Since the STK Engine does not support this type of analysis, an external function was created to take data from STK and use it to analyze a store-and-forward based communication system.

In order to analyze this type of system, three Time Ordered Access (TOA) reports from STK are required. A sample TOA report is shown in Figure 21. Once the reports have been generated by STK and sent to Matlab, the relaytime.m function searches through the Target-ToConstellation chain, Constellation-to-Constellation chain and Constellation-to-Ground chain TOA reports in order to find all the valid relays and compute the constellation performance.

Chain-Target_2_Sats

Strand Name
Target/Beijing_China To Satellite/CS10
Target/Beijing_China To Satellite/CS10
Target/Beijing_China To Satellite/CS10
Target/Beijing_China To Satellite/CS10
Target/Beijing_China To Satellite/CS10
Target/Beijing_China To Satellite/CS10
Target/Beijing_China To Satellite/CS10

\begin{tabular}{l} 
Start Time (UTCG) \\
\hdashline 20 Nov 2011 08:00:00.000 \\
20 Nov 2011 09:29:01.403 \\
20 Nov $2011 \quad 11: 10: 52.573$ \\
20 Nov $2011 \quad 21: 47: 48.964$ \\
20 Nov $2011 \quad 23: 27: 52.986$ \\
21 Nov $201101: 10: 36.779$ \\
21 Nov $2011 \quad 07: 34: 05.818$
\end{tabular}

政

166.647
919.406
767.554
864.574
875.884
383.210
486.029

Figure 21. A sample TOA report between the Target and CubeSat constellation

It is important to note in the sample report that the location of the target name, under the "Strand Name" category, will always start at the eighth character and the name of the CubeSat is always only four characters long.

\section{$\underline{\text { Search Routine }}$}

In order to determine the performance of a CubeSat constellation, an accurate and robust timekeeping routine needed to be created. The purpose of the relaytime.m function is to keep 
track of which CubeSats have received a data package, and the earliest time that the data package was received. Using the timekeeping data, the time it takes to relay a signal from the target to a ground station can be determined.

The relaytime.m function requires several inputs in order to properly run. The first inputs are the three TOA reports from STK that will be used to determine which CubeSats receive data and at what time. The number of CubeSats in the constellation is also needed in order to create a matrix to keep track of times and which CubeSats have received the data. Finally, the constraint of how long it takes for communication to be established and for data to be transmitted, referred to as acquisition time, is needed to determine the time at which each CubeSat has completed the transfer and received the data.

The first thing the function checks for is to make sure that access exists between the target and CubeSat constellation and between the constellation and a ground station. If one or the other does not exist, then there is no possible way for data to be relayed and the function notifies the user and returns "NaN" for all variables. If it has been determined that there is access between the target and constellation and the constellation and a ground station then the function initializes several tracking matrices. The first matrix to be initialized is the relay_time matrix which is responsible for storing all the valid relay times. Since it is unknown how many successful relays there will be for a given scenario, the size of this matrix is dependent on how many times the constellation has access to the target and ground stations. For an MxN matrix, $\mathrm{M}$ would represent how many accesses the constellation has with the ground stations, and $\mathrm{N}$ represents how many accesses there are between the target and constellation. In order to accurately calculate the minimum, maximum and average relay time, this matrix is initialized with "NaN" in every element. This prevents Matlab from automatically placing a " 0 " in empty 
cells that do not correspond to a valid relay, which would skew the average relay time towards “0”.

After the relay_time matrix has been initialized, three vectors are set to zero in order to count the number of data acquisitions, passes and deliveries for each CubeSat. The length of these vectors is $1 \mathrm{xN}$ where $\mathrm{N}$ is the number of CubeSats in the constellation. Each time a CubeSat successfully acquires data from the target, passes the data to another CubeSat or delivers the data to a ground station, the corresponding count for that CubeSat is increased by one.

The last two matrices that are initialized are sat_check and time_check. The sat_check matrix is initialized with " 0 " in every element and is responsible for keeping track of which CubeSats have received data and which ones have not. This is accomplished by having the matrix store a "1", or "True", if the CubeSat has received data or a "0", or "False", if it has not. The time_check matrix is responsible for keeping track of the earliest time that each CubeSat receives a data package. Every time there is an access between two objects, the time stored in this matrix is checked to ensure that the earliest time is stored, if not then the time is redefined with the new, earlier time. The time_check matrix is initialized with "NaN" in every element to avoid problems when comparing times. The size of both the sat_check and time_check matrices is determined by how many CubeSats are in the constellation, and how many accesses exist between the target and constellation. Given an $\mathrm{MxN}$ matrix, $\mathrm{M}$ would be the number of accesses between the target and constellation, and $\mathrm{N}$ would be the number of CubeSats in the constellation.

Once all matrices and counts have been initialized the search for valid relays can begin. In order to build an accurate sat_check and time_check matrix, each access in the TOA reports must be checked one by one. The search begins with the Target-To-Constellation report and the 
first access between the target and CubeSat constellation. The start time of the relay is

determined by the start time of the access between the target and constellation which is stored in a "start" variable, the element in the sat_check matrix corresponding to the CubeSat that received the data is set to " 1 " and the corresponding element in the acquisition count vector is increased by one.

The next step is to search through the Constellation-To-Constellation report in order to find the earliest time that each CubeSat receives the data. In order to accomplish this, the function goes line by line checking the name of the CubeSats involved in the current access with the sat_check matrix. A "change" variable is used to track if any changes to the time_check matrix have occurred while searching through the report. If a successful access is found and changes one of the earliest time values in the time_check matrix, the change variable is set to "1", or true. When a CubeSat that has received the data (i.e. sat_check matrix element is a "1") is involved in an access with another CubeSat, the time of that access is checked against the time_check matrix. If the earliest time that the CubeSat that has the data is later than the time of the access between the two CubeSats, then it is not a valid access and the function continues to the next access in the report. If the earliest time that the CubeSat that has the data is earlier than the time of the access between the two CubeSats then it is considered a valid access. When there is a valid access between two CubeSats both corresponding elements in the sat_check matrix are set to " 1 ", the earliest time is updated in the time_check matrix and the corresponding elements in the pass count vector are increased by " 1 ". This continues until every access in the Constellation-To-Constellation has been checked. If the change variable was set to a "1" while going through the report, then the function will go search through the Constellation-ToConstellation report again, finding the earliest time each CubeSat receives the data until there is no change. At the end of this search, the sat_check matrix will have a record of which CubeSats 
received data and the time_check matrix will contain the earliest time that each CubeSat received the data.

Once the earliest time that each CubeSat has received the data is known a search through the third TOA report, between the constellation and ground stations, is performed to determine the first time that a ground station receives the data from a CubeSat. The process is exactly the same as in the first search; checking to ensure the CubeSat that has access to the ground station has received the data before the access time with the ground station. The first time that a CubeSat, carrying data, accesses a ground station the search stops and stores the access time in the "stop" variable and the corresponding element in the delivery count vector is increased by one. Only the first successful access between the constellation and ground station is stored since it will be the earliest time that the ground station can receive the data. Once a "start" time and "stop" time have been determined, the difference is taken to find the relay time, which is then stored in the relay_time matrix. After successfully finding a relay time, the whole process is repeated with the second access between the target and constellation and continues until all accesses in the Target-To-Constellation report are analyzed.

Three sample reports are shown in Figure 22, which will serve as an example of how the relaytime.m function works. 


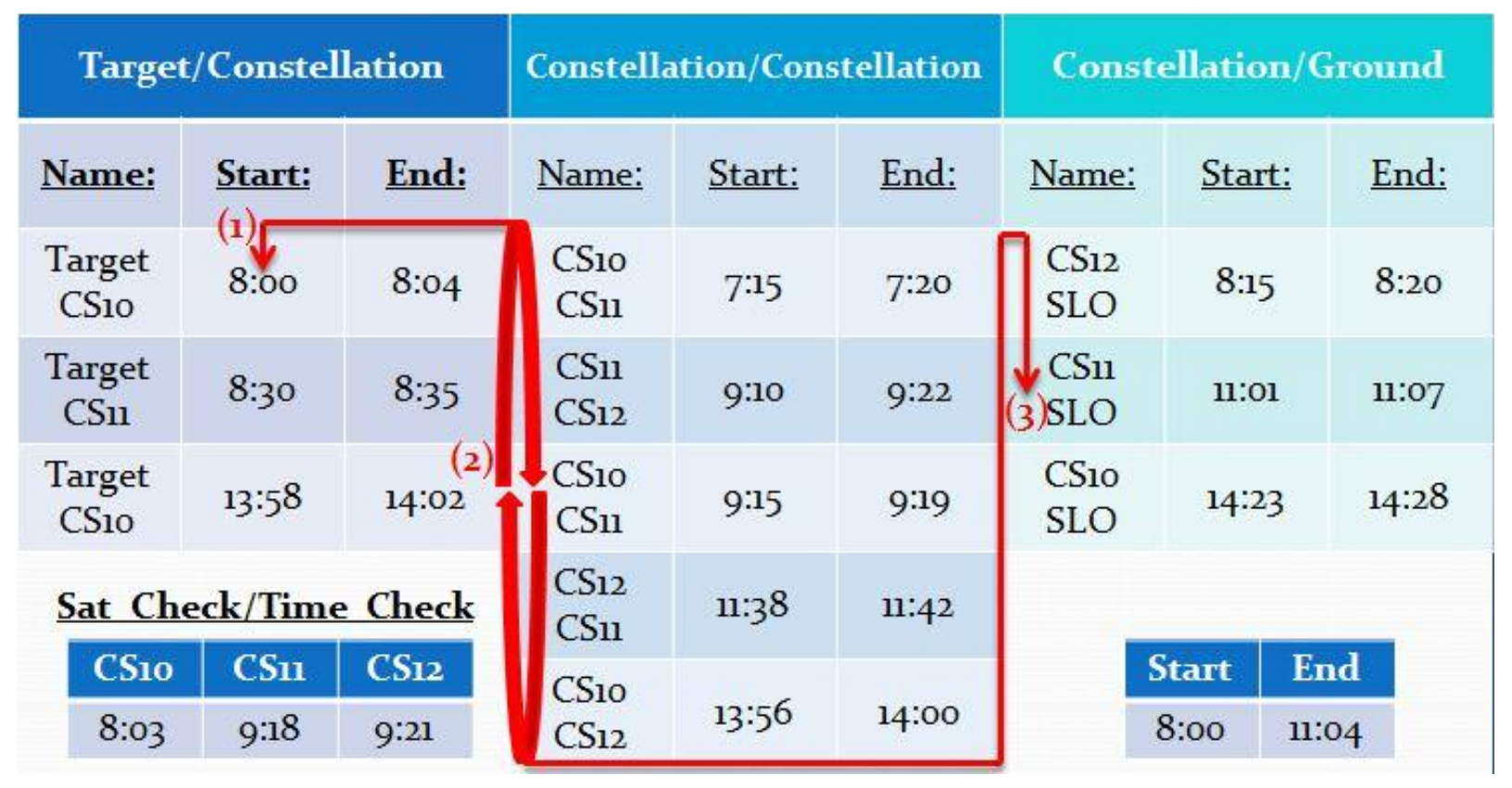

Figure 22. relaytime.m search routine example

The relaytime.m function will start with the first access in the "Target/Constellation" report, find the earliest time that each CubeSat receives the data using the Constellation/Constellation report and finally find the first time that a CubeSat with the data can deliver it to a ground station using the Constellation/Ground report. Assuming a three minute acquisition and data transfer time, the relay starts at 8:00 (1) from the access between Target/CS10, shown in the "Start" box in the lower right corner, with CS10 receiving the entire data package at 8:03, shown in the "CS10" box in the lower right corner. The function then enters a "while-loop" (2) searching for the earliest time that each CubeSat receives the data. The first access between CS10/CS11 occurs before CS10 has received the data, so it is an invalid access. The second access between CS11/CS12 is invalid because currently only CS10 has received the data. The third access between CS10/CS11 is valid since CS10 has received the data and the time is after when CS10 first receives the data. So at 9:15 the data is relayed from CS10 to CS11, and at 9:18, CS11 has successfully acquired the data. The next access between CS12/CS11 is valid since CS11 now has the data, and the access time between the two is after CS11 has received the data, so CS12 
receives the data at 11:41. Since there have been changes made to when each satellite receives

the signal, another search is done through the Constellation/Constellation. The first access is still invalid due to the early time; however the second access between CS11/CS12, which was invalid before due to the fact that neither had received the data, is now a valid access because the earliest time that CS11 receives the data is at 9:18 which is during the access between CS11/CS12 and the remaining time from 9:18 to the end of the access at 9:22 is long enough for CS11 to relay the data to CS12. So the earliest time for CS12 will be updated from 11:41 to 9:21. Since another change was made to the time at which each CubeSat receives the data, the search continues through the Constellation/Constellation report until no change is found. Once the function is done searching through the Constellation/Constellation report, the earliest possible time that each CubeSat receives the data for the first access between Target/Constellation is known. Finally, a search is done through the Constellation/Ground report (3) to find the first time that a CubeSat can deliver the data. The first access between CS12/SLO is invalid since it occurs before CS12 has received the data. The second access between CS11/SLO is a valid access since it is after CS11 received the data. The end time of 11:04 is stored in the "End" box in the lower right corner and the difference between the "Start" and "End" times is used to find the relay time.

At the end of the analysis the relay_time matrix contains all the valid relay times that were found. Using these values, the minimum, maximum and average times are calculated and returned to the user in the results box inside SATCAT.

\section{$\underline{\text { Limitations and Assumptions }}$}

Since the relaytime.m function requires three TOA reports corresponding to the communication chains between the target and constellation, constellation and constellation, and constellation and ground stations, it is not possible to analyze the performance of a single 
CubeSat without a target. Under normal circumstances, a CubeSat does not have a specific target on the ground, rather continuously taking data that will need to be down-linked to a ground station. In order to analyze the performance of a single CubeSat, an arbitrary target will need to be added to provide the system with a "start" time for data acquisition.

Additionally, each of the TOA reports is based strictly on line-of-sight between objects. In order to communicate based on line-of-sight it is assumed that each CubeSat has an omnidirectional antenna, capable of communicating with any object in its view. Likewise, it is assumed that the target has an omni-directional antenna as well, removing the need for the target to track any CubeSats passing overhead. 


\section{QuakeSat Example}

\section{$\underline{\text { QuakeSat Introduction }}$}

QuakeSat is a joint partnership between Stanford University and QuakeFinder, a private research organization focused on creating a system for forecasting major earthquakes, to investigate predictive earthquake technologies. Using an innovative space based technique QuakeSat will search for the emission of Extremely Low Frequency (ELF) magnetic signals, which have been shown to be an earthquake precursor phenomenon. ${ }^{7}$ The mission objectives for QuakeSat include the ability to detect, record and downlink earthquake ELF emission data and demonstrate that the CubeSat design is a cost-effective platform for conducting significant space science research experiments. As previously discussed, one of the major limitations in CubeSat technology is the long periods of time between communication between the CubeSat and ground station. When a payload, such as QuakeSat's, is collecting data that needs to be analyzed on the ground very shortly after being acquired this is a major problem. In order for earthquake prediction to be useful, the data needs to be available to users on the ground as soon as possible; however, current CubeSat technology requires waiting for the vehicle to pass over a user controlled ground station which could be hours after the data was originally collected.

In order to investigate the usefulness of a CubeSat constellation for data relaying several scenarios will be created and their relay performance compared. First, current CubeSat technologies will be investigated by analyzing the performance of QuakeSat as a single vehicle. Second, the performance of a constellation consisting of QuakeSat and two relay CubeSats will be investigated. And finally, a constellation consisting of QuakeSat and 36 relay CubeSats will be analyzed. A 2D map displaying the orbital ground tracks for each scenario can be seen in 
Figure 23 - Figure 25.

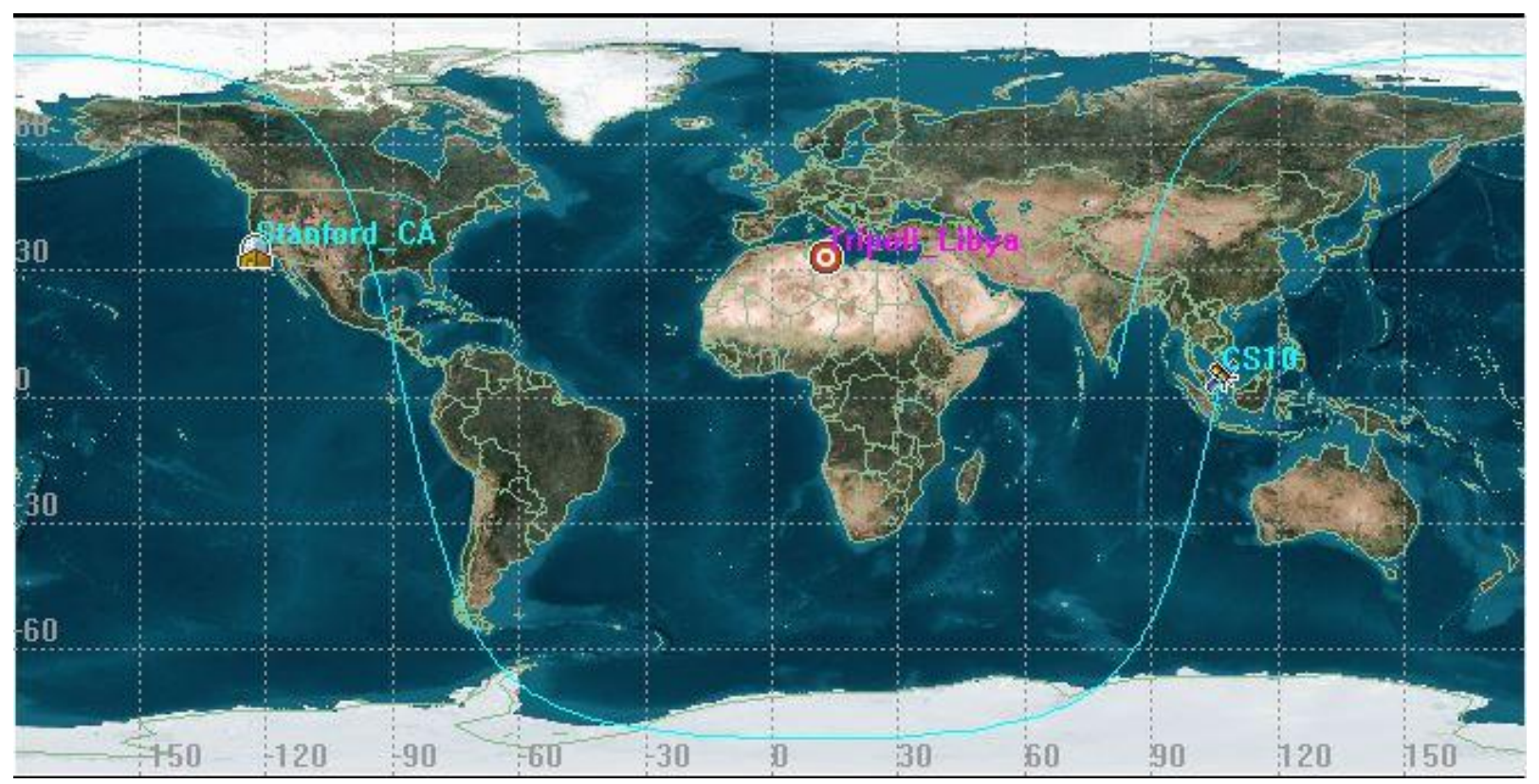

Figure 23. 2D map depicting the QuakeSat scenario

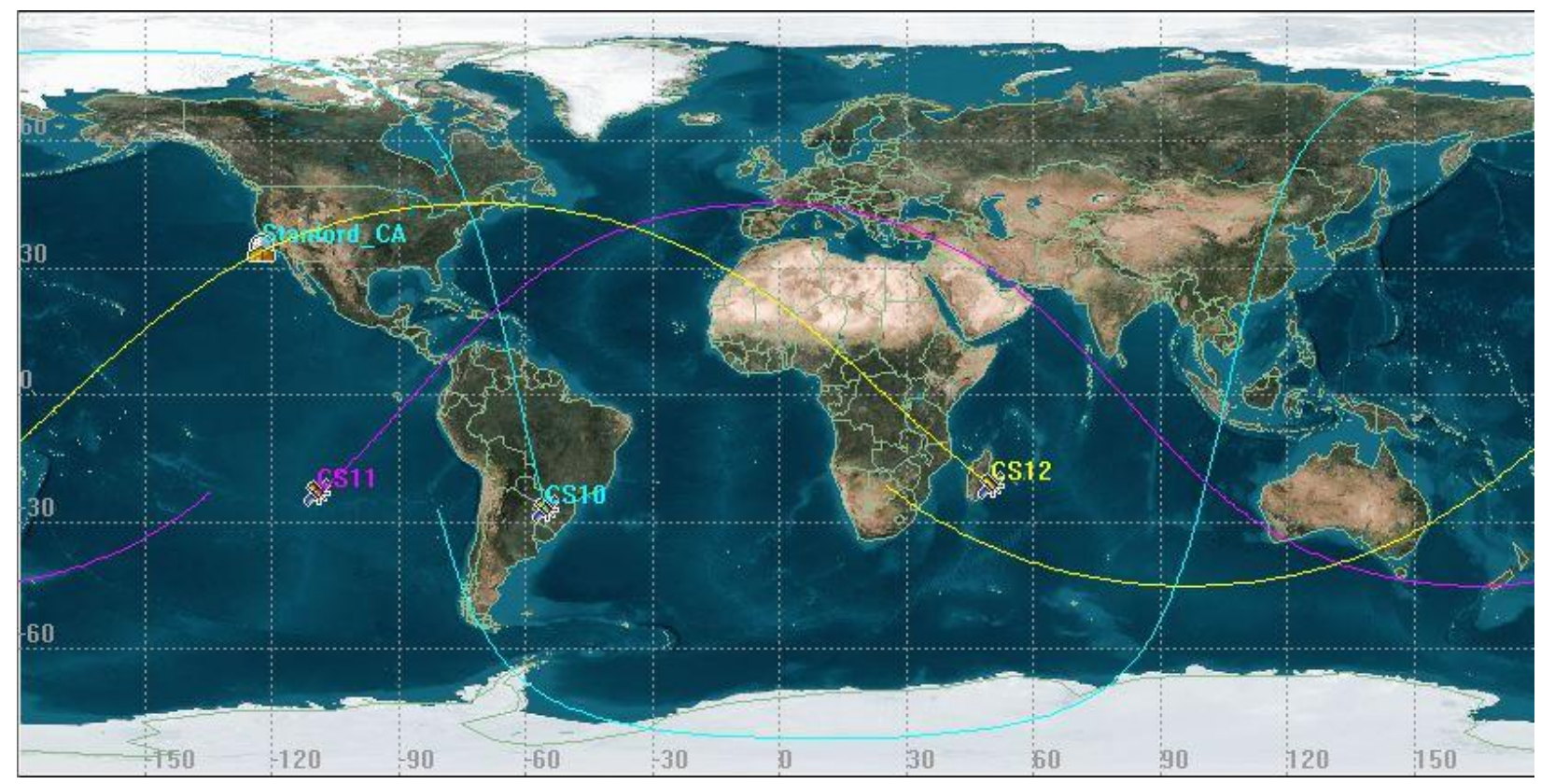

Figure 24. 2D map depicting the QuakeSat + 2 relay CubeSats scenario 


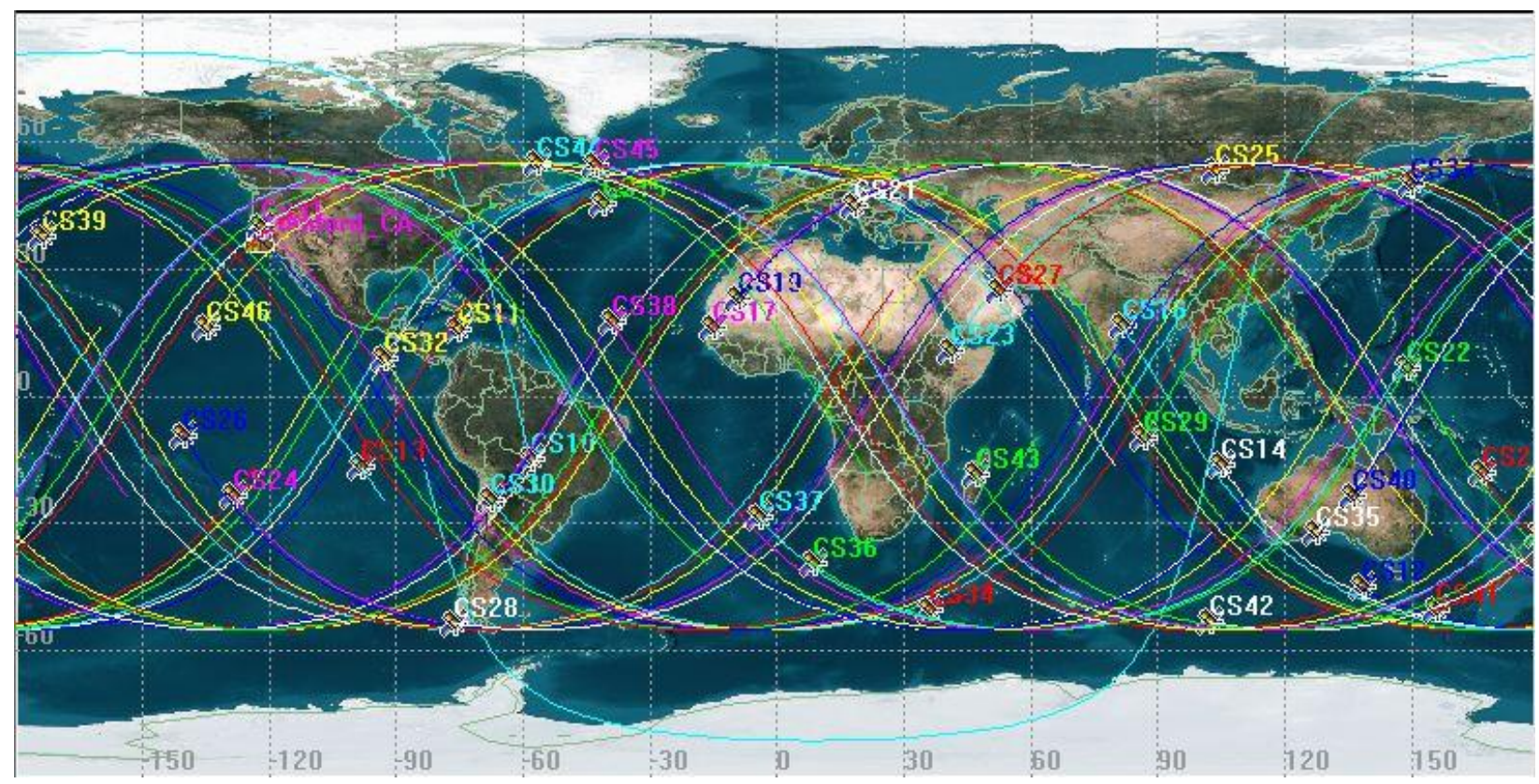

Figure 25. 2D map depicting the QuakeSat + 36 CubeSat relay scenario

Each scenario will be analyzed for twenty-four hours and their performances compared. One important aspect of analyzing a defined constellation, especially with CubeSats which don't usually have the ability to maintain their position in an orbit, is to compare the current and future performance as the constellation changes over time.

\section{$\underline{\text { Assumptions }}$}

Several assumptions were made in order to perform the constellation analysis. The first is that it takes three minutes to acquire and transfer data between two objects. The second is the each CubeSat was able to be placed at a specific initial location which would require many launches over several years and precise orbital placement. All previously mentioned assumptions regarding communication between objects were also used in this analysis.

\section{QuakeSat Performance}

When looking at the performance of QuakeSat on its own it is important to remember the limitation of the relaytime.m function. In order to analyze a single CubeSat it is required to have 
a target as well. For this analysis, Tripoli, Libya was chosen for its location roughly $180^{\circ}$ longitude from Stanford, Ca, where the ground station is located. The relay performance for a twenty-four hour period is shown in Table 2.

Table 2. QuakeSat relay time results

\begin{tabular}{|c|c|}
\hline Minimum Time: & 31 minutes, 53 seconds \\
\hline Maximum Time: & 511 minutes, 41 seconds \\
\hline Average Time: & 331 minutes, 18 seconds \\
\hline Number of Relays: & 6 \\
\hline
\end{tabular}

When trying to predict earthquakes, an average time of 328 minutes can make your data obsolete. In order to understand the distribution of relay times a histogram, shown in Figure 26, can be used and it is found that out of the 6 accesses with the ground station in the twenty-four hour period, the majority of relay times are above the 300 minute mark.

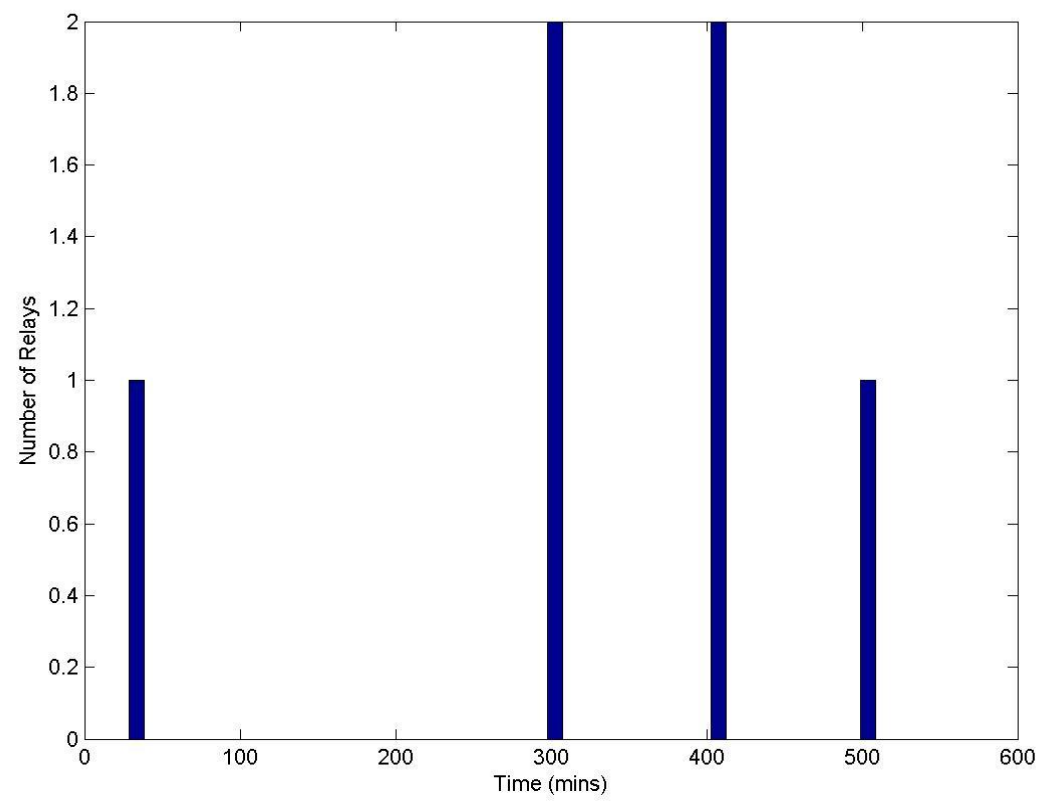

Figure 26. Relay time histogram for QuakeSat scenario

\section{QuakeSat +2 Relay Performance}

By adding two relay CubeSats, the target at Tripoli, Libya is no longer needed; Instead, QuakeSat will become the target for the CubeSat constellation. The two relay CubeSats were 
placed into $800 \mathrm{~km}$ circular orbit one with an inclination of $45^{\circ}$ and the other with an inclination of $135^{\circ}$. The performance results of the twenty-four hour period are shown in Table 3.

Table 3. QuakeSat + 2 Relay CubeSats results

\begin{tabular}{|c|c|}
\hline Minimum Time: & 6 minutes \\
\hline Maximum Time: & 497 minutes, 56 seconds \\
\hline Average Time: & 152 minutes, 43 seconds \\
\hline Number of Relays: & 36 \\
\hline
\end{tabular}

It can be seen that simply by providing QuakeSat with two relay CubeSats, the minimum time drastically reduces, while the maximum and average times shorten slightly. The other important thing to note is that the total number of relays in a twenty-four hour period increased from 6 to 36 with the addition of the two relays. This is an improvement on a single CubeSat, but for certain applications, like that of QuakeSat, an average time of 149 minutes can still make the data outdated by the time it reaches the ground station. Again, by looking at histogram, shown in Figure 27, the user can determine the distribution of relay times for the scenario. For this constellation, it can be seen that more than half of the relays take less than 100 minutes to complete, which is again an improvement from QuakeSat alone. 


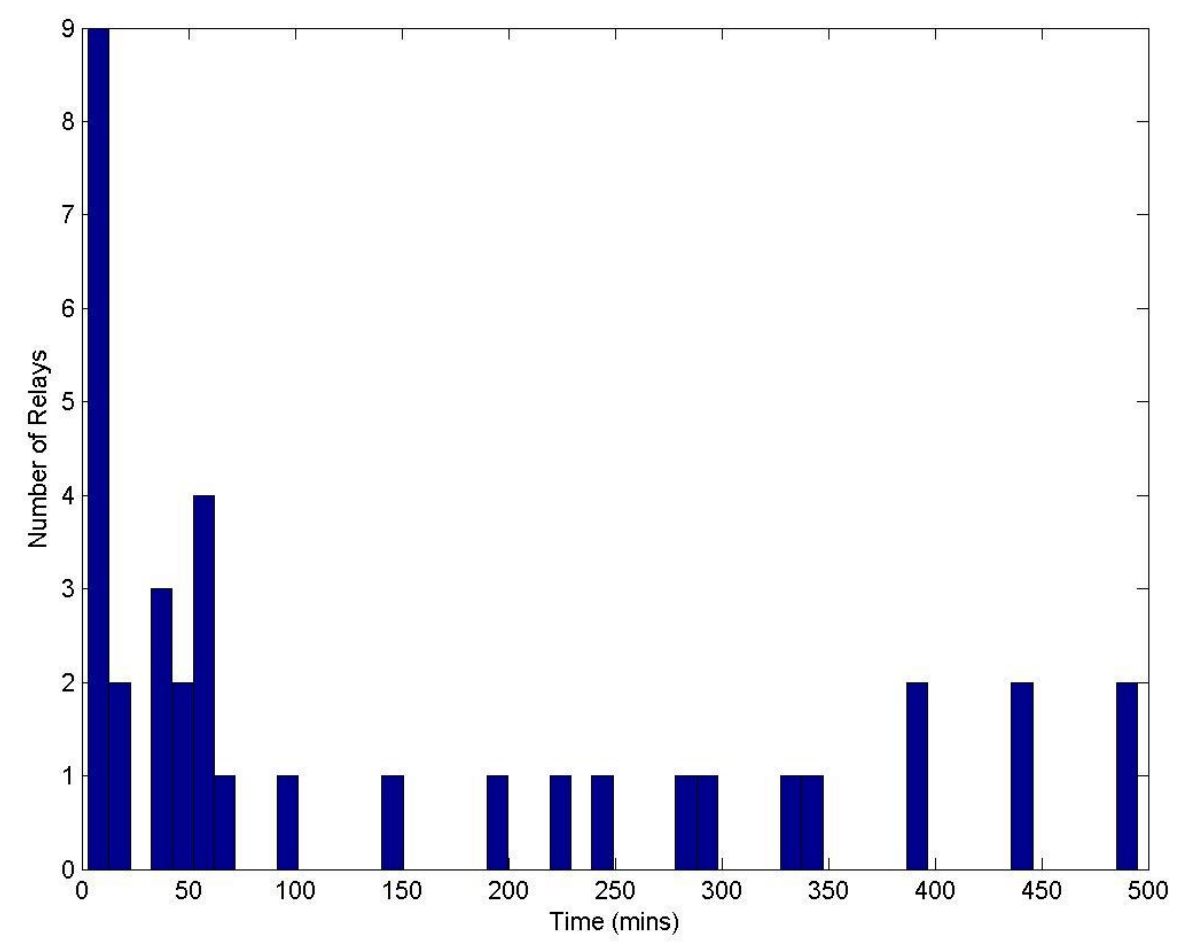

Figure 27. Relay time histogram for QuakeSat + 2 Relays

Now that there is more than one CubeSat in the constellation, the constellation performance data can be analyzed. The number of times each CubeSat receives data, passes data to another CubeSat and delivers data to the ground station is shown in Figure 28.
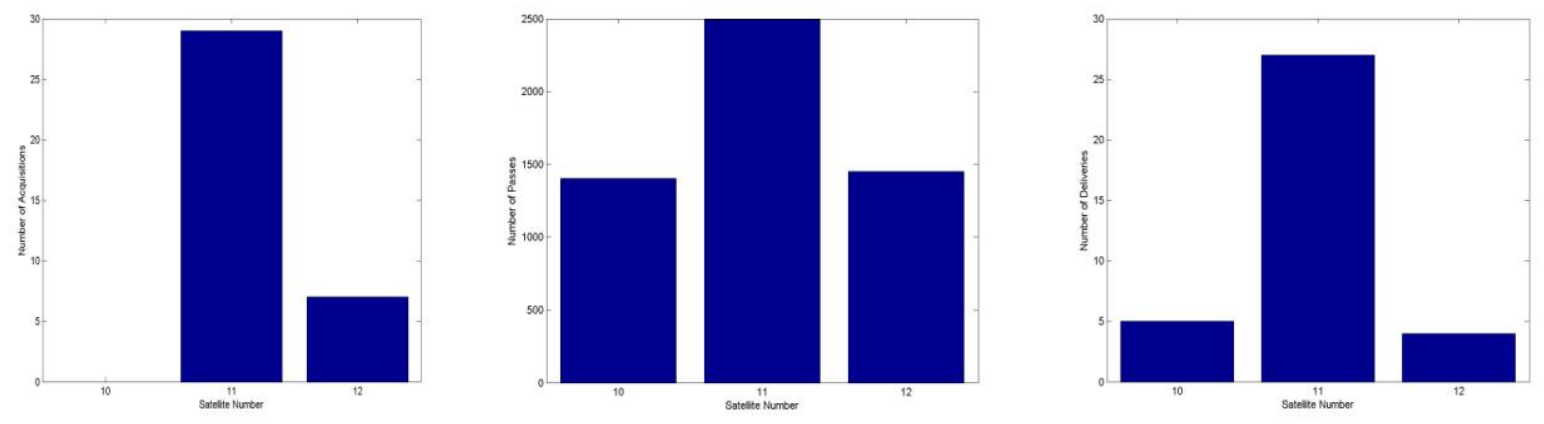

Figure 28. (Left to Right) The number of acquisitions, passes, and deliveries per CubeSat

Since CubeSat 10, corresponding to QuakeSat, is the target it never acquires the data due to the fact that it is the source of the data. However, it is able to pass and deliver the data to the other CubeSats and the ground station. 


\section{QuakeSat + 2 Relay Future Performance}

While the current performance of QuakeSat was improved by adding two relay CubeSats, the performance of the same constellation a year in the future could be quite different. With drag, solar radiation pressure and $3^{\text {rd }}$ body effects acting on each CubeSat, their positions relative to each other could change drastically over time and therefore change the performance of the constellation. Using the "Change Analysis Time Period" feature of SATCAT, the constellation was propagated one year into the future and the performance re-calculated with the results shown in Table 4.

Table 4. QuakeSat + 2 Relays results one year in the future

\begin{tabular}{|c|c|}
\hline Minimum Time: & 6 minutes \\
\hline Maximum Time: & 82 minutes, 38 seconds \\
\hline Average Time: & 50 minutes, 58 seconds \\
\hline Number of Relays: & 14 \\
\hline
\end{tabular}

As can be seen, this particular constellation's performance increases a year after the initial analysis. Both the maximum and average times have been reduced significantly, but at the cost of over half the total number of accesses. By analyzing this constellation in the present and in the future, it is shown that the performance is not consistent, but is an improvement over QuakeSat by itself.

\section{$\underline{\text { QuakeSat + Constellation Performance }}$}

In order to increase the performance even more, a constellation of 36 CubeSats, plus QuakeSat will be analyzed. The constellation is formed by placing six $55^{\circ}$ inclination orbits with each orbit separated by $60^{\circ}$ in Right Ascension of the Ascending Node (RAAN). Within each orbit are six CubeSats which are separated by $60^{\circ}$ in True Anomaly (TA). The altitude of each CubeSat in each orbit ranges from $799 \mathrm{~km}$ to $801 \mathrm{~km}$. This separation in altitude is used to 
create drifting between CubeSats, hopefully creating a more realistic orbital distribution. The results of this constellation are shown in Table 5.

Table 5. QuakeSat + Constellation relay time results

\begin{tabular}{|c|c|}
\hline Minimum Time: & 3 minutes \\
\hline Maximum Time: & 11 minutes, 45 seconds \\
\hline Average Time: & 3 minutes, 9 seconds \\
\hline Number of Relays: & 564 \\
\hline
\end{tabular}

Obviously, the performance of this constellation greatly exceeds both QuakeSat alone and QuakeSat with two relays. With a minimum time of 3 minutes this is considered instantaneous communication between QuakeSat and the ground station. The only reason the minimum time is 3 minutes is due to the initial assumption that it takes 3 minutes for acquisition and data transfer. An average time of slightly over 3 minutes provides the system response needed for an earthquake prediction mission. Verification of the fast relay times can be seen in the histogram shown in Figure 29.

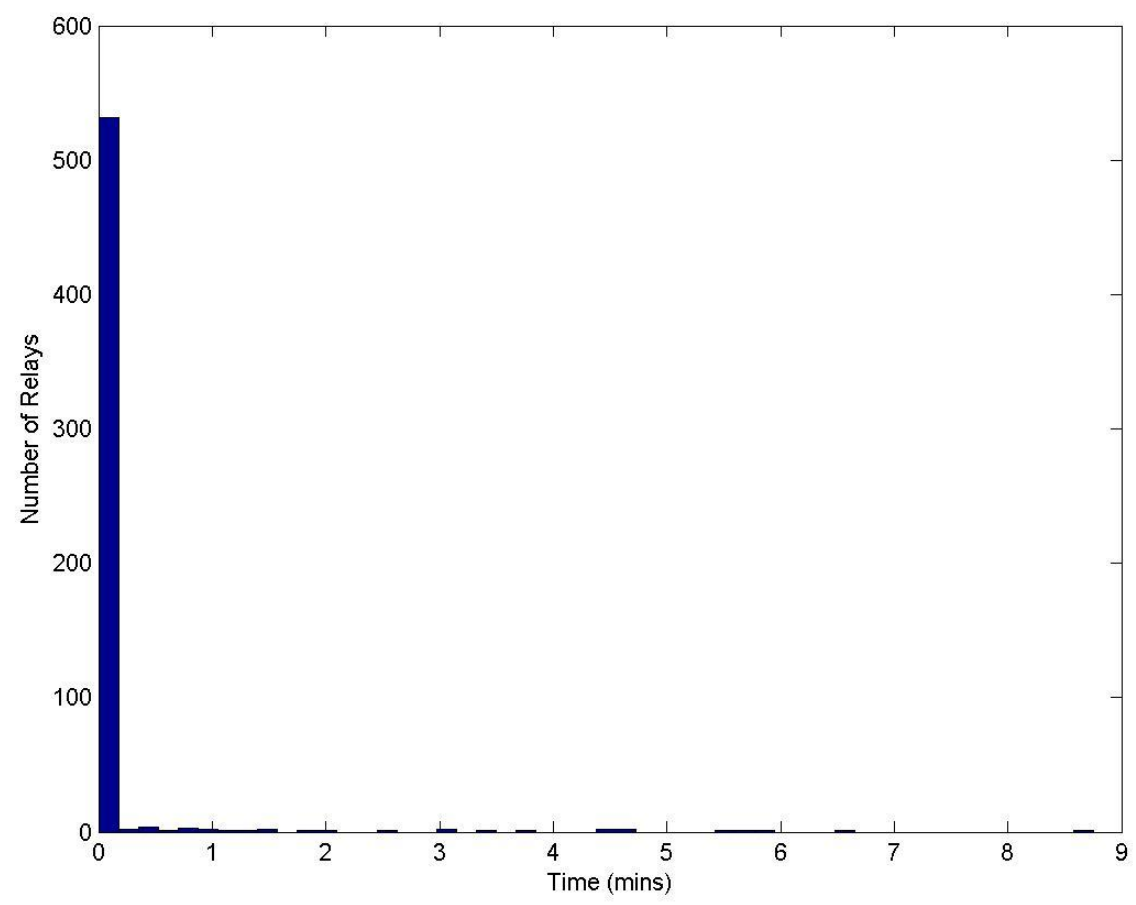

Figure 29. QuakeSat + Constellation relay time histogram 
With over 500 accesses between the constellation and ground station in a twenty-four hour period, commands can be uplinked and data down-linked much more frequently than in the previous scenarios. With a constellation of this size, it is also important to investigate the performance of individual CubeSats in the constellation. The acquisition, pass and delivery counts, shown in Figure 30, allow the user to visualize which CubeSats are contributing to the performance of the constellation more than others.
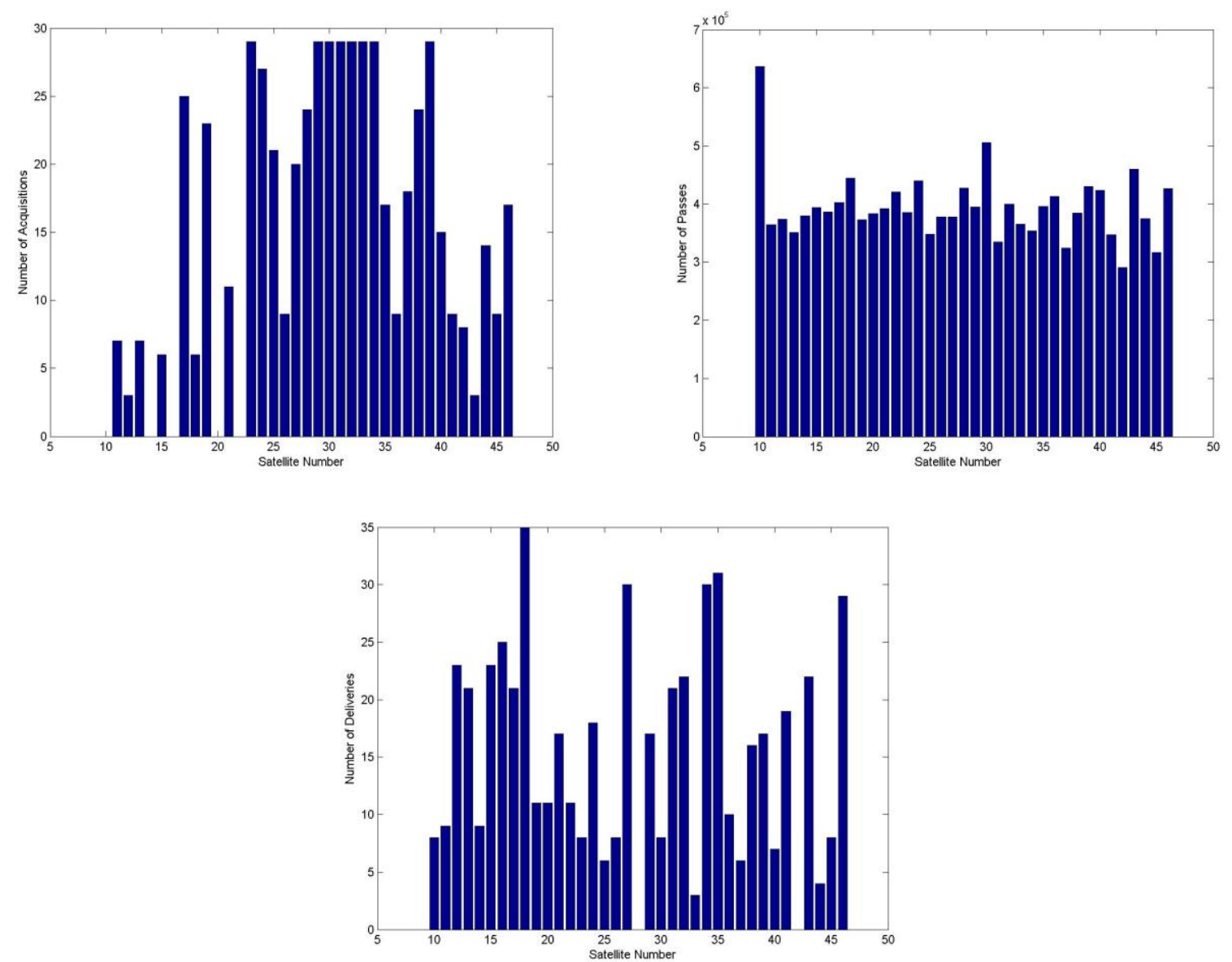

Figure 30. (Left to Right) Acquisition, delivery and pass counts for QuakeSat + Constellation

By inspecting these plots, it can be seen that a few CubeSats in the constellation never acquire data from the target or deliver the data to the ground station; however, every CubeSat participates in the data passing process. Further analysis would need to be performed to determine the performance of the constellation if the lower performing CubeSats were removed. 


\section{QuakeSat + Constellation Future Performance}

With a constellation as large and dependent on orbital placement as this, it is important to look at the performance over time. Using SATCAT, the analysis period for the constellation was change to a year later and the performance re-calculated. The results, shown in Table 6, confirm that while the performance changes slightly, it still provides near instantaneous data relay.

Table 6. QuakeSat + Constellation relay times a year later

\begin{tabular}{|c|c|}
\hline Minimum Time: & 3 minutes \\
\hline Maximum Time: & 17 minutes, 6 seconds \\
\hline Average Time: & 3 minutes, 20 seconds \\
\hline Number of Relays: & 652 \\
\hline
\end{tabular}

After a year of perturbations, the performance of individual CubeSats in the constellation changes slightly, as shown in Figure 31.
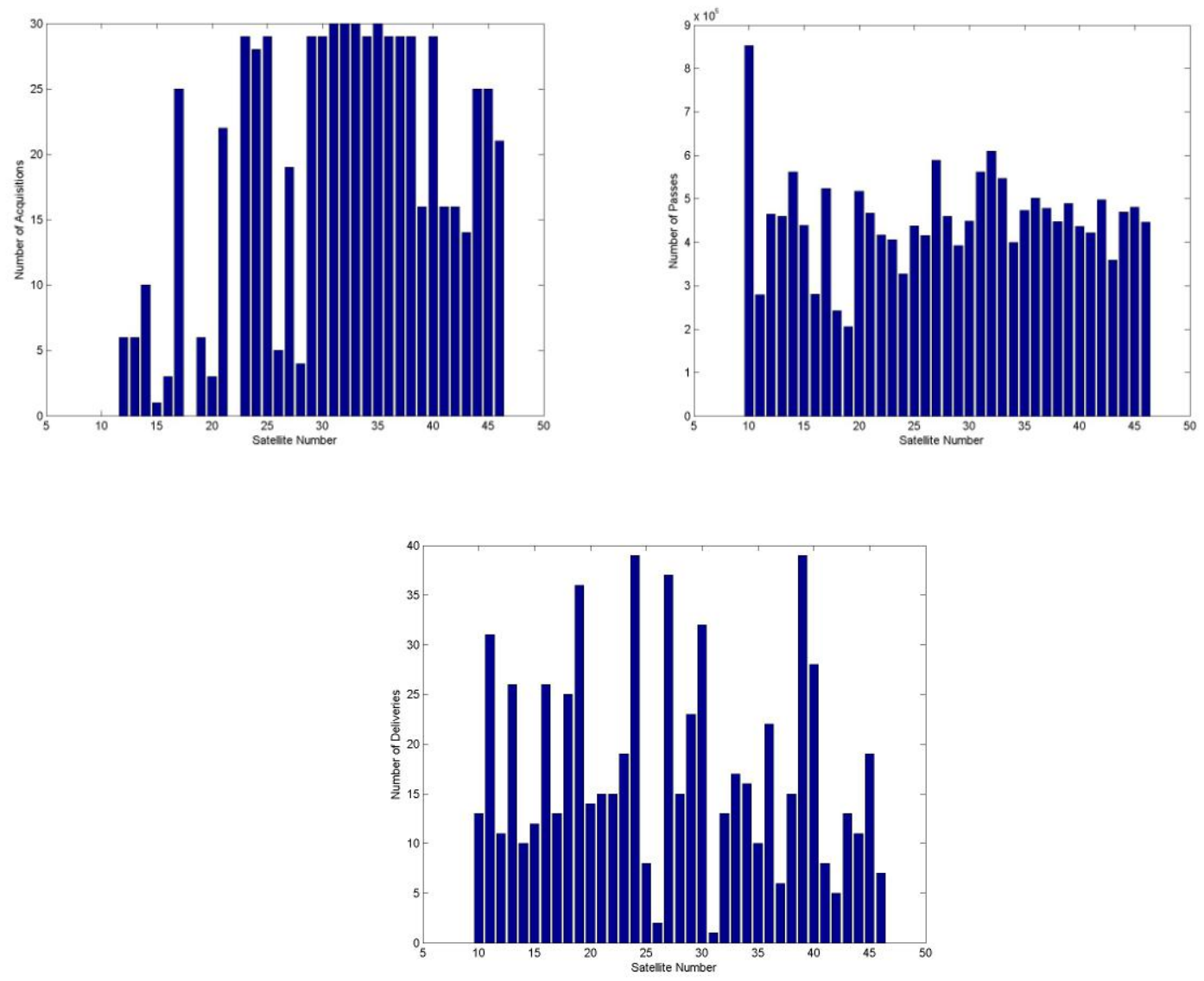

Figure 31. (Left to Right) Acquisition, delivery and pass counts for each CubeSat 


\section{Comparison}

By comparing the performance of each scenario, shown in Table 7, it is easy to see that a constellation of CubeSats, regardless of size, can increase the number of access and reduce the communication latency between the ground station and CubeSat.

Table 7. Comparison of relay performance

\begin{tabular}{|cccccc|}
$\begin{array}{c}\text { Time } \\
\text { (mins) }\end{array}$ & QuakeSat & $\begin{array}{c}\text { QuakeSat } \\
+2\end{array}$ & $\begin{array}{c}\text { QuakeSat } \\
+2 \\
\text { Year Later }\end{array}$ & $\begin{array}{c}\text { QuakeSat }+ \\
\text { Constellation }\end{array}$ & $\begin{array}{c}\text { QuakeSat }+ \\
\text { Constellation } \\
\text { Year Later }\end{array}$ \\
\hline Minimum: & $28: 53$ & $6: 00$ & $6: 00$ & $3: 00$ & $3: 00$ \\
\hline Maximum: & $508: 41$ & $497: 56$ & $82: 38$ & $11: 45$ & $17: 06$ \\
\hline Average: & $328: 18$ & $152: 43$ & $50: 58$ & $3: 09$ & $3: 20$ \\
\hline Relays: & 6 & 36 & 14 & 564 & 652 \\
\hline
\end{tabular}

While the combination of different orbits, number of satellites, number of ground stations and position of ground stations is nearly infinite, these scenarios aim to show that increasing the performance of a CubeSat is possible. 


\section{Conclusions}

By using the spatial and temporal analysis features of STK, coupled with the STK/Matlab interface a robust tool was created to analyze the performance of CubeSat constellations based on a store-and-forward communications model which is not currently supported by the STK Engine. Utilizing the Connect messaging format through a socket connection on the local machine, a Matlab graphical user interface, called SATCAT, was constructed in order to provide the user with the ability to control many aspects of the STK Engine externally. Functions such as creating a new scenario, populating the scenario with objects, setting constraints and creating reports are all available inside SATCAT, bypassing the STK interface completely.

Controlling STK externally, while cumbersome, provides the ability to create automated design schemes, or custom analysis tools. In the case of this project, the STK Engine does not support the ability to analyze a communication system based on a store-and-forward model. A custom function needed to be designed to take access reports from STK and calculate the performance of a CubeSat constellation. The resulting function is able to use a Time Ordered Access (TOA) report from STK to find how long it would take for data to be relayed from a target to a ground station through a network of CubeSats. In addition to calculating the relay time, the function also generates statistical information on the performance of the constellation as well as a ground access report that can be used to track CubeSats from the ground stations.

Three sample scenarios were created to demonstrate the use and performance analysis capabilities of SATCAT. The performance of a single CubeSat was analyzed and compared to the performance of a three CubeSat constellation and a thirty-seven CubeSat constellation. The results showed that using a constellation of CubeSats greatly reduced the time required to relay data from one location to another, while also increasing the total number of accesses between the CubeSats and ground stations. 
Since the relay time function is separate from the SATCAT graphical user interface, future users can take advantage of the external control provided by SATCAT and integrate their own custom function without needing to create a new interface between STK and Matlab. This allows SATCAT to be a modular tool, much like STK is a modular software program allowing many different add-ons to be used. 


\section{Improvements}

One of the biggest areas for improvement is the fidelity of determining access between two objects. Currently access is based directly off of line-of-sight, instead of actual CubeSat communication abilities. One of STK's additional modules is the Communications package. STK/Communications allows users to define and analyze detailed communications systems, generate detailed link budget reports and graphs, and incorporate detailed rain models, atmospheric losses and RF interference sources. ${ }^{8}$ Receiver and transmitter models can be attached to targets, ground stations and satellites allowing for a more detailed communication link. Using STK/Communications will allow all typical link parameters to be analyzed including frequency, signal-to-noise ratio, Bit Error Rate and gain. Another benefit to using STK/Communications is the extensive antenna types supported. Users would be able to use predefined antennas such as dipole, helix or horn antennas, or create a custom antenna that fits the system requirements. By integrating the STK/Communications package with the current storeand-forward analysis technique provided by SATCAT, a much more detailed and accurate system can be modeled and analyzed.

In addition to using the STK/Communications package, more control over individual object constraints would allow for more flexibility and better modeling of real-world scenarios. Currently constraints are placed on entire constellations instead of individual objects in the constellation. This would provide the user the ability to add specific constraints to each object in the scenario, providing a more accurate model.

Besides improving the fidelity of the access computation between objects, other areas of improvement include modifying SATCAT to be more of a generic interface between STK and Matlab. Currently, SATCAT is designed for the purpose of analyzing CubeSat constellations and displaying the performance characteristics. If SATCAT was made into a general interface 
between STK and Matlab, then future users could use it to perform design optimization, or automated computations by augmenting their custom function with the generic interface. Additionally, greater control of object constraints would allow the user to define custom constraints for each object, rather than entire constellations. This would provide the ability to better model different CubeSats that may be included in a constellation. Finally, having a maximum number of ninety CubeSat limits the possibility of analyzing larger constellations. With some modification, SATCAT should be able to handle an unlimited number of CubeSats in a constellation. 


\section{System Requirements}

The STK/Matlab interface used in SATCAT requires initial setup and product licenses. In order to use this software, a STK/Matlab interface license and a STK/Connect license is required. Both licenses are usually included in the Professional or Expert version of STK. In addition to the STK licenses, a 32-bit version of Matlab 6.1, or higher, must be installed on the same machine. The STK/Matlab interface currently does not support 64-bit versions of Matlab. Once STK and a 32-bit version of Matlab are installed on the user's machine, the initial setup for the STK/Matlab interface must be completed. The process for establishing the necessary links between STK and Matlab are as follows: ${ }^{9}$

1) Ensure Matlab and STK are closed

2) Go to your STK Matlab Toolbox (C:Program Files\AGISSTK STK_Version\Matlab\ToolboxLocal); the path may differ depending on your version of STK

3) Copy the following files:
a. agiCleanPath.m
b. agiGetConfig.m
c. agiInit.m
d. agiResetConfig.m
e. stk.m
f. stkInit.m

4) Paste the files into your local Matlab Toolbox

5) Open Matlab and type: agiInit('setup'). The STK/Matlab Interface Configuration window will appear. Click Continue.

6) Next you'll be prompted to locate the AGI M-files. Go to:

C:Program Files\AGIISTK_Version\Matlab and click OK. 
a) If this folder does not exist, the Matlab option was not selected during installation of STK. You must repeat the installation and choose Modify to add the required STK/Matlab files and folders.

7) Next you'll be prompted to locate the AGI MEX-files. Go to:

C:Program Files\AGISTK_Version \bin and click OK. Select the file of interest, mexConnect.dll.

8) Now we'll direct Matlab to an STK host, which may or may not be on the same machine as where you run Matlab. Click Yes to configure a default MexConnect Host.

9) Select the default MexConnect host (machine name and TCP/IP port of the machine). localhost:5001 assumes that STK is running on the same machine as Matlab and the 5001 is the STK default value for the TCP/IP port.

10) Now click Yes to configure STK/Connect initialization file.

11) Browse to C:IProgram Files\AGIISTK_Version\Connect\PCData and click on the file named Connect.dat.

12) When prompted to configure a STKHome folder, click No. (STK will not prompt you for this.)

\section{Setup Confirmation}

1) With Matlab still open, Startup STK with no scenario. At the Matlab prompt type stkInit to initialize a connection to STK. If everything is in order you should see the following response:

>> stkInit

initializing STK/Matlab Interface with AGIDefault preferences 
Warning: mexConnect: Connecting to localhost:5001

2) Now assign a number to the socket connection (the resulting number may differ):

> stkOpen

ans $=$

2

3) Alternately, you can assign a variable to hold that value, so you can generically call the variable in an M-file.

$>$ conid $=$ stkOpen;

4) Create an STK scenario using the assigned socket number and the New Scenario Connect command (substitute the 2 with another number, or the variable 'conid' if necessary). >> stkExec(2,'New / Scenario Scenario1')

5) STK now has created Scenario1.

If you would like access to the Matlab .m files, please contact California Polytechnic State University - San Luis Obispo’s Aerospace Engineering Department at:

Aerospace Engineering Department

Building 41A, Room 134

Cal Poly State University

1 Grand Avenue

San Luis Obispo, CA 93407

Phone: 805-756-2562

aero@calpoly.edu 


\section{$\underline{\text { References }}$}

\footnotetext{
1 "About Us." CubeSat in the News. Web. 21 Nov. 2011. <http://www.cubesat.org/index.php/about-us>.

${ }^{2}$ Crook, Matthew R. "NPS CUBESAT LAUNCHER DESIGN, PROCESS AND REQUIREMENTS." Thesis. Naval Postgraduate School Monterey, CA 93943-5000, 2009. Defense Technical Information Center. Naval Postgraduate School Monterey, CA 93943-5000, June 2009. Web. 21 Nov. 2011. <http://www.dtic.mil/cgibin/GetTRDoc?AD=ADA501503\&Location=U2\&doc=GetTRDoc.pdf >.

${ }^{3}$ Analytical Graphics, Inc. (AGI), Analysis Software for Land, Sea, Air, and Space. Web. 21 Nov. 2011. $<$ http://www.agi.com/>.

4 "AGI Technology Basics - Analytical Graphics, Inc." Analytical Graphics, Inc. (AGI), Analysis Software for Land, Sea, Air, and Space. Web. 21 Nov. 2011. <http://www.agi.com/products/agi-technology-basics/>.

5 "Object Model." Analytical Graphics, Inc. (AGI), Analysis Software for Land, Sea, Air, and Space. Web. 21 Nov. 2011. <http://www.agi.com/resources/help/online/STKDevKit/source/automationtree/objmodel.htm>.

${ }^{6}$ Matlab - The Language of Technical Computing. Web. 21 Nov. 2011.

$<$ http://www.mathworks.com/products/matlab/>.

7 "QuakeSat Nano-Satellite Stanford University Space Systems Development Laboratory."QuakeFinder. Web. 21 Nov. 2011. <http://www.quakefinder.com/services/quakesat-ssite/>.

8 "STK/Communications - Analytical Graphics, Inc." Analytical Graphics, Inc. (AGI), Analysis Software for Land, Sea, Air, and Space. Web. 21 Nov. 2011. <http://www.agi.com/products/by-product-type/applications/stk/add-onmodules/stk-communications/>.

9 "STK/Matlab Interface - STK -- API - Connect - AGI Forum." Analytical Graphics, Inc. (AGI), Analysis Software for Land, Sea, Air, and Space. 4 Feb. 2008. Web. 21 Nov. 2011.

$<$ http://www.agi.com/agiforum/messages.aspx?TopiclD=28>.
} 